\title{
Transformations of Spherical Blocks
}

\author{
Amir-Kian Kashani-Poor and Jan Troost \\ Laboratoire de Physique Théorique, ${ }^{1}$ Ecole Normale Supérieure, \\ 24 rue Lhomond, 75005 Paris, France \\ E-mail: kashani@lpt.ens.fr, troost@lpt.ens.fr
}

ABSTRACT: We further explore the correspondence between $\mathcal{N}=2$ supersymmetric $\mathrm{SU}(2)$ gauge theory with four flavors on $\epsilon$-deformed backgrounds and conformal field theory, with an emphasis on the $\epsilon$-expansion of the partition function natural from a topological string theory point of view. Solving an appropriate null vector decoupling equation in the semi-classical limit allows us to express the instanton partition function as a series in quasi-modular forms of the group $\Gamma(2)$, with the expected symmetry $W\left(D_{4}\right) \rtimes S_{3}$. In the presence of an elementary surface operator, this symmetry is enhanced to an action of $W\left(D_{4}^{(1)}\right) \rtimes S_{4}$ on the instanton partition function, as we demonstrate via the link between the null vector decoupling equation and the quantum Painlevé VI equation.

Keywords: Conformal and W Symmetry, Supersymmetric gauge theory, Topological Strings

ARXIV EPRINT: 1305.7408

\footnotetext{
${ }^{1}$ Unité Mixte du CNRS et de l'Ecole Normale Supérieure associée à l'Université Pierre et Marie Curie 6, UMR 8549.
} 


\section{Contents}

1 Introduction 1

2 The two sides of the correspondence $\quad 3$

2.1 The four-point conformal block on the sphere 3

2.2 The $\mathcal{N}=2$ supersymmetric $\mathrm{SU}(2)$ theory with four flavors 5

$\begin{array}{lll}2.3 & \text { The parameter map } & 7\end{array}$

$3 \quad$ The null vector decoupling equation $\quad 7$

3.1 The null vector decoupling equation in spherical coordinates 8

3.2 Symmetries of the null vector decoupling equation 9

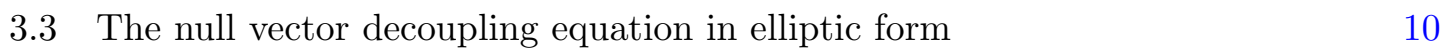

$\begin{array}{ll}3.4 \text { The monodromy } & 11\end{array}$

4 The genus expansion $\quad 12$

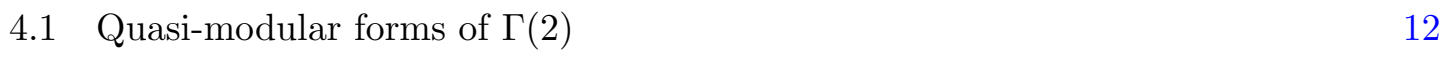

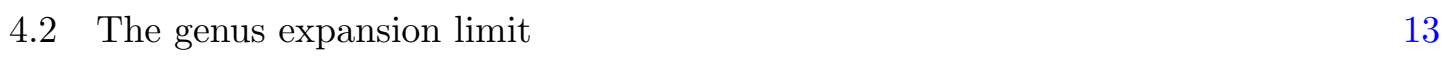

$\begin{array}{ll}\text { 4.3 The elliptic recursion relation } & 14\end{array}$

4.4 Solving the null vector decoupling equation in the genus expansion 16

5 Conclusion $\quad 20$

A The null vector decoupling equation in elliptic variables $\quad 21$

$\begin{array}{ll}\text { A.1 The change of variables } & 21\end{array}$

A.2 Properties of elliptic and modular functions 23

B The monodromies of shifted Weierstrass integrands 25

$\begin{array}{ll}\text { C The group action } & 26\end{array}$

\section{Introduction}

The correspondence between $\epsilon$-deformed $\mathcal{N}=2$ supersymmetric gauge theories in four dimensions and two-dimensional conformal field theories [1] provides a fascinating bridge between two highly developed domains of theoretical physics. The bridge allows for twoway beneficial exchange. In this paper, in the spirit of $[2,3]$ and especially [4], we further explore the ramifications of this correspondence in the case of the gauge theory with four fundamental flavors, which yields the spherical four-point conformal block.

Our main interest on the gauge theory side of the correspondence is the instanton partition function in an expansion in the topological string coupling $g_{s}$ and the deformation 
parameter $s$. This generalization of the genus expansion naturally arises upon computation via geometric engineering and the topological string. In conformal field theory, it corresponds to the limit in which both the central charge $c$ and the exchanged weight $h$ become large, with $h \gg c$. In this limit, it proves advantageous to express the spherical conformal block in elliptic variables [5], in particular replacing the cross ratio $x$ of the four insertion points via a modular parameter $q_{2}$. The elliptic recursion relation which the spherical fourpoint conformal block satisfies permits a simple proof that the genus expansion has zero radius of convergence. The main motivations for studying it nevertheless are threefold: it arises in the semi-classical limit of the conformal block, the coefficients of the expansion are quasi-modular forms, ${ }^{1}$ and the expansion is natural from the point of view of topological string theory. Understanding the physical importance of the quasi-modular symmetry from the point of view of conformal field theory, and drawing lessons for the non-perturbative formulation of the topological string, remain important open challenges.

Our path towards the computation of the conformal block in a genus expansion proceeds via the null vector decoupling equation satisfied by the four-point correlator with an additional insertion of a degenerate operator. After the passage to elliptic variables [5], the computation proceeds in close analogy to our treatment of $\mathcal{N}=2^{*}$ gauge theory in [4]. In particular, the $\tau$-derivatives of the expansion coefficients are manifestly quasi-modular forms, now of the modular subgroup $\Gamma(2)$ rather than $\Gamma(1)$, and we again observe experimentally that they also integrate to quasi-modular forms. The $S$-duality and flavor symmetry of the $N_{f}=4$ theory, $W\left(D_{4}\right) \rtimes \mathrm{SL}(2, \mathbb{Z})$, as identified in [6], is manifest in our expressions. $^{2}$

The gauge theory interpretation of the four-point conformal block with an additional degenerate insertion has been proposed in [10]: it is conjectured to reproduce the instanton partition function in the presence of an elementary surface operator. We will demonstrate that this equation (before taking the semi-classical limit) essentially coincides with the quantum Painlevé VI equation [11-13]. The Painlevé VI equation is the most general (rational) ordinary second order differential equation with no movable singularities except poles [14-16]. Its quantization can be performed by reformulating the equation in terms of a first order Hamiltonian system, then quantizing the Hamiltonian and studying the corresponding Schrödinger equation. The symmetries of the resulting equation have been extensively studied in [17]. By mapping this discussion to our variables, we deduce the action of the extended symmetry group $W\left(D_{4}^{(1)}\right) \rtimes S_{4}$ on the instanton partition function in the presence of the surface operator.

The organization of this paper is as follows. In section 2, we review the spherical four-point conformal block and the natural elliptic variables corresponding to the problem. On the gauge theory side, we review how the symmetry group $W\left(D_{4}\right) \rtimes \operatorname{SL}(2, \mathbb{Z})$ arises in

\footnotetext{
${ }^{1}$ More precisely, the genus expansion of the logarithm of the conformal block yields coefficients that are quasi-modular forms up to the constant (i.e. $q_{2}$-independent) terms. The latter are furnished by the contribution of the three-point function of Liouville theory to the correlator, identified in [1] as yielding the perturbative part of the Nekrasov partition function.

${ }^{2}$ This symmetry can also be analyzed via Coulomb integral formulae in conformal field theory, as in [7], based on results in $[8,9]$.
} 
$N_{f}=4$ Seiberg-Witten theory. Section 3 identifies the null vector decoupling equation, which governs the instanton partition function in the presence of an elementary surface operator, with the quantized Painlevé VI equation and discusses the symmetry group $W\left(D_{4}^{(1)}\right) \rtimes S_{4}$ of this equation. We then review the mapping of this equation to elliptic variables, with many details of the derivation provided in appendix A. The semi-classical limit of the null vector decoupling equation is treated in section 4 . There, we discuss the proof of non-convergence of the genus expansion based on the elliptic recursion relation for the conformal block. We also demonstrate that under the assumption of quasi-modularity, this relation allows for a computation of the corresponding topological string amplitudes in the field theory limit. Finally, we turn to the solution of the null vector decoupling equation in the semi-classical limit, which intrinsically gives rise to quasi-modular results for these amplitudes in the $\epsilon_{2} \rightarrow 0$ limit.

\section{The two sides of the correspondence}

In this section, we will review the four-point spherical conformal block and $\epsilon$-deformed $\mathcal{N}=2$ supersymmetric Yang-Mills theory with $\mathrm{SU}(2)$ gauge group and four flavors. The correspondence [1] equates the former with the instanton partition function of the latter.

\subsection{The four-point conformal block on the sphere}

In this subsection, we wish to review the notion of the four-point conformal block on the sphere, and explain how elliptic variables arise naturally in this context. We consider the four-point correlator

$$
C_{4}=\left\langle V_{h_{1}}\left(z_{1}\right) V_{h_{2}}\left(z_{2}\right) V_{h_{3}}\left(z_{3}\right) V_{h_{4}}\left(z_{4}\right)\right\rangle
$$

on the sphere of Virasoro primary fields $V_{h_{i}}\left(z_{i}\right)$ of weight $h_{i}$. The cross ratio of the four insertion points,

$$
x=\frac{\left(z_{3}-z_{4}\right)\left(z_{2}-z_{1}\right)}{\left(z_{3}-z_{1}\right)\left(z_{2}-z_{4}\right)}
$$

is a conformal invariant that serves as a coordinate on the complex structure moduli space $\mathcal{M}_{4,0}$ of the four-punctured sphere. The conformal transformation

$$
z^{\prime}=\frac{\left(z-z_{4}\right)\left(z_{2}-z_{1}\right)}{\left(z-z_{1}\right)\left(z_{2}-z_{4}\right)}
$$

maps the points $\left(z_{1}, z_{2}, z_{3}, z_{4}\right)$ to the points $(\infty, 1, x, 0)$. Conformal invariance determines the $z_{i}$ dependence of the four-point correlator $C_{4}$ up to a function of the cross ratio,

$$
\begin{aligned}
C_{4} & =\left|\frac{\left(z_{4}-z_{1}\right)\left(z_{2}-z_{1}\right)}{z_{2}-z_{4}}\right|^{2 \sum h_{i}} \prod_{i \neq 1}\left|z_{i}-z_{1}\right|^{-4 h_{i}} \lim _{z \rightarrow z_{1}}\left|z-z_{1}\right|^{-4 h_{1}}\left\langle V_{h_{1}}\left(z^{\prime}(z)\right) V_{h_{2}}(1) V_{h_{3}}(x) V_{h_{4}}(0)\right\rangle \\
& =\left|\frac{\left(z_{4}-z_{1}\right)\left(z_{2}-z_{1}\right)}{z_{2}-z_{4}}\right|^{2 \sum h_{i}-4 h_{1}} \prod_{i \neq 1}\left|z_{i}-z_{1}\right|^{-4 h_{i}} G_{1234}(x) .
\end{aligned}
$$

We have here introduced the matrix element $G_{1234}$ via

$$
G_{1234}(x)=\lim _{z \rightarrow z_{1}} \mid\left(\left.z^{\prime}(z)\right|^{4 h_{1}}\left\langle V_{h_{1}}\left(z^{\prime}(z)\right) V_{h_{2}}(1) V_{h_{3}}(x) V_{h_{4}}(0)\right\rangle=\left\langle h_{1}\left|V_{h_{2}}(1) V_{h_{3}}(x)\right| h_{4}\right\rangle,\right.
$$


with $\left|h_{4}\right\rangle$ and $\left\langle h_{1}\right|$ defined with regard to the $z^{\prime}$ coordinate system. This matrix element in turn can be expressed as

$$
G_{1234}(x)=\sum_{h} C_{12 h} C_{34}^{h} \mathcal{F}_{14}^{23}(h \mid x) \overline{\mathcal{F}}_{14}^{23}(h \mid \bar{x})
$$

in terms of the conformal blocks $\mathcal{F}_{14}^{23}(h \mid x)$. By expressing $G_{1234}(x)$ in this fashion, we have separated the contributions which are determined completely by the Virasoro algebra, encoded in the conformal blocks, from the content specific to a given conformal field theory, encoded in the three point functions $C_{i j}^{k}$. The sum is over the conformal families that appear both in the operator product expansion of $V_{h_{3}}$ and $V_{h_{4}}$, and of $V_{h_{1}}$ and $V_{h_{2}}$. We call these conformal families intermediate, with an associated intermediate highest weight.

The matrix element $G_{1234}$ is related to the four-point correlator with insertions at positions $z_{i}$ via the coordinate transformation (2.3). A different choice of coordinate transformation permutes the assignment of the points $z_{i}$ to the points $\{0,1, \infty\}$. It acts on the cross ratio $x$ via a rational transformation, and establishes equalities (so-called channel dualities) between the various functions $G_{i j k l}(x)$ with permutations of the indices and rational transformations of the argument $x$, as follows:

$$
G_{1234}(x)=G_{1432}(1-x)=\frac{1}{|x|^{4 h_{3}}} G_{4231}(1 / x) .
$$

Via equation (2.6), this yields identities for conformal blocks, after summation over the respective intermediate momenta.

The conformal blocks are functions of the cross ratio $x$, which serves as a representative of the 4-tuple $\left(z_{1}, z_{2}, z_{3}, z_{4}\right)$ under the equivalence relation of conformal transformations. We can associate an elliptic curve to the equivalence class of this tuple via the equation

$$
y^{2}-t(t-1)(t-x)=0,
$$

with 2 -torsion points lying at $(t, y)=(0,0),(1,0),(x, 0) \cdot{ }^{3}$ Choosing the first two as a basis in the group of 2-torsion points, this defines a map from equivalence classes of four insertion points on a sphere to the equivalence classes of elliptic curves together with a basis of their group of 2-torsion points. It is not hard to show that the latter is isomorphic to $\mathbb{H} / \Gamma(2)$, the upper half plane $\mathbb{H}$ modded out by the group $\Gamma(2)$ of matrices in $\operatorname{SL}(2, \mathbb{Z})$ which are equal to the identity modulo $2 .{ }^{4}$ Conversely, given an elliptic curve with modular parameter $\tau$ together with such a basis, we can define a map to the class of four tuples via

$$
\left(z_{1}, z_{2}, z_{3}, z_{4}\right)=\left(\infty, \wp\left(\frac{1}{2}\right), \wp\left(\frac{\tau}{2}+\frac{1}{2}\right), \wp\left(\frac{\tau}{2}\right)\right)
$$

by invoking the Weierstrass $\wp$-function. The invariant cross ratio of this tuple is

$$
x=\frac{e_{3}-e_{2}}{e_{3}-e_{1}},
$$

\footnotetext{
${ }^{3}$ To see this, transform this equation into Weierstrass form by eliminating the quadratic term in $t$ via the substitution $t^{\prime}=t-\frac{x+1}{3}$ and correctly normalizing the cubic term via $y^{\prime}=2 y$. The equation is hence solved by $\left(t^{\prime}, y^{\prime}\right)=\left(\wp(z), \wp^{\prime}(z)\right)$, and the zeros of $\wp^{\prime}(z)$ clearly lie at $\wp(z)=-\frac{x+1}{3}, 1-\frac{x+1}{3}, x-\frac{x+1}{3}$.

${ }^{4} \mathbb{H} / \operatorname{SL}(2, \mathbb{Z})$ describes equivalence classes of elliptic curves. Modding out by the smaller group $\Gamma(2)$ avoids identifying tori whose 2-torsion points are permuted.
} 
where we have introduced the standard nomenclature for the half-periods of $\wp$, see equation (A.22). The cross ratio $x$ is invariant under the action of $\Gamma(2)$ on $\tau$. We have thus established the isomorphism between classes of 4 -tuples on the sphere and $\mathbb{H} / \Gamma(2)$. We can hence equally well express the conformal blocks $\mathcal{F}_{14}^{23}(h \mid x)$ as functions of $[\tau] \in \mathbb{H} / \Gamma(2)$. The permutation of the points $z_{i}$, which acts as a rational transformation on the cross ratio $x$, acts via an element of $\mathrm{SL}(2, \mathbb{Z}) / \Gamma(2)$ on $\tau$. The action of $\Gamma(2)$ on $\tau$ gives rise to additional symmetries not accessible when the blocks are expressed as functions of $x$. These will play a central role in section 4 .

When treating the five point function on the sphere in the next section, it will prove convenient to extend this isomorphism to a (necessarily multi-valued) map from the sphere to the torus which maps the four insertion points $(\infty, 1, x, 0)$ on one sheet to the 2 -torsion points. The fifth insertion point is then parameterized by an elliptic variable.

\subsection{The $\mathcal{N}=2$ supersymmetric $\mathrm{SU}(2)$ theory with four flavors}

On the four-dimensional side of the two-dimensional/four-dimensional correspondence, we mostly consider a gauge theory observable that has several incarnations. It was introduced in the course of the computation of the prepotential of $\mathcal{N}=2$ gauge theories via instanton calculus. As the integrals that arise in this computation are divergent, one can consider their equivariant counterpart $Z_{\text {inst }}\left(\epsilon_{1}, \epsilon_{2}\right)$, introducing the two parameters $\epsilon_{1}$ and $\epsilon_{2}$ [18]. In this incarnation, the partition function $Z_{\text {inst }}$ is defined as a formal sum in the instanton counting parameter $x=q_{U V}$ corresponding to the $U V$ coupling. The partition sum $Z_{\text {inst }}$ can also be obtained from the topological string partition function with the appropriate target space geometry to engineer the field theory. Via the holomorphic anomaly equations, this definition gives rise to all order results in the instanton counting parameter, order by order in the topological string coupling squared $g_{s}^{2}=\epsilon_{1} \epsilon_{2}$ and the deformation parameter $s=\left(\epsilon_{1}+\epsilon_{2}\right)^{2}$.

We concentrate on the $\mathrm{SU}(2)$ gauge theory with $\mathcal{N}=2$ supersymmetry and $N_{f}=4$ flavors, which is superconformal in the massless limit. The space of marginal deformations of the theory can be parameterized by a parameter $\tau$ taking values in the upper half plane. The flavor symmetry is enhanced from $\mathrm{SU}(4)$ to $\mathrm{SO}(8)$. Since this feature is important to us, we will review how it arises.

An $\mathcal{N}=2$ theory with $N_{f}$ massless hypermultiplets $\left(Q_{i}, \tilde{Q}_{i}\right)$, such that $Q_{i}$ transform in the fundamental and $\tilde{Q}_{i}$ in the anti-fundamental of the gauge group, generically enjoys $\mathrm{SU}\left(\mathrm{N}_{\mathrm{f}}\right)$ flavor symmetry. The flavor symmetry is enhanced to $\mathrm{SO}\left(2 \mathrm{~N}_{\mathrm{f}}\right)$ when the hypermultiplets transform in a pseudo-real representation of the gauge group, such as the fundamental representation for the gauge group SU(2). A pseudo-real representation exhibits an antisymmetric intertwiner $R$ which relates the generators $\sigma_{a}$ within the representation to their complex conjugates, via $\sigma_{a}^{*}=-R^{-1} \sigma_{a} R$. The kinetic terms of the theory therefore exhibit $\mathrm{SU}\left(2 \mathrm{~N}_{\mathrm{f}}\right)$ symmetry, made manifest by introducing the field $\tilde{Q}_{f}=R \tilde{Q}$. The $\mathcal{N}=2$ superpotential term $\tilde{Q} \Phi Q$, with $\Phi$ the chiral superfield in the $\mathcal{N}=2$ vector multiplet, can be rewritten as

$$
\left(Q^{T} \tilde{Q}_{f}^{T}\right)\left(\begin{array}{ll}
0 & 1 \\
1 & 0
\end{array}\right) \Phi R^{-1}\left(\begin{array}{c}
Q \\
\tilde{Q}_{f}
\end{array}\right) .
$$


This term hence breaks the flavor symmetry from $\mathrm{SU}\left(2 \mathrm{~N}_{\mathrm{f}}\right)$ to $\mathrm{SO}\left(2 \mathrm{~N}_{\mathrm{f}}\right)$. Note that a real representation, i.e. a symmetric intertwiner $R$, would have required the matrix $\left(\begin{array}{cc}0 & 1 \\ -1 & 0\end{array}\right)$ instead, breaking the flavor symmetry to $\operatorname{Sp}\left(2 \mathrm{~N}_{\mathrm{f}}\right)$. Returning to the gauge group $\mathrm{SU}(2)$ with $N_{f}=4$ fundamental flavors, the flavor symmetry is thus enhanced from SU(4) to $\mathrm{SO}(8)$, as announced.

A mass term consistent with $\mathcal{N}=2$ supersymmetry is provided by the superpotential term

$$
m_{i} \tilde{Q}_{i} Q_{i}=\left(Q_{i}^{T} \tilde{Q}_{f, i}^{T}\right)\left(\begin{array}{cc}
0 & m_{i} \\
-m_{i} & 0
\end{array}\right) R^{-1}\left(\begin{array}{c}
Q_{i} \\
\tilde{Q}_{f, i}
\end{array}\right)
$$

Note that the mass matrix takes values in a Cartan of $\mathfrak{s o}(8)=D_{4}$. We can expand it in terms of the standard basis $\left\{H_{i}\right\}$ for the Cartan subalgebra as $M=\sum_{i} m_{i} H_{i}$. The mass matrix breaks the flavor symmetry to $\mathrm{SO}(2)^{4}$. The Weyl group $W\left(D_{4}\right)$ of the $D_{4}$ Lie algebra can be identified as the subgroup of $\mathrm{SO}(8)$ which maps the Cartan of $D_{4}$ to itself under conjugation. The vector $\left(m_{1}, \ldots, m_{4}\right)$ hence furnishes a representation of this group, which acts on it via permutations and an even number of sign changes. This action on the parameters $m_{i}$ is therefore a symmetry of the theory.

The maximal regular subalgebra $\mathrm{SU}(2)^{4} \subset \mathrm{SO}(8)$ will play a distinguished role in the following. Given the basis vectors $\epsilon_{i}$ of root space dual to the generators $H_{i}$ of the Cartan introduced above, a convenient choice for the four roots of $D_{4}$ corresponding to these four $\mathfrak{s u}(2)$ subalgebras is

$$
\alpha_{0}=-\epsilon_{1}-\epsilon_{2}, \quad \alpha_{1}=\epsilon_{1}-\epsilon_{2}, \quad \alpha_{3}=\epsilon_{3}-\epsilon_{4}, \quad \alpha_{4}=\epsilon_{3}+\epsilon_{4} .
$$

We can express the mass matrix $M$ in terms of the basis $E_{i}^{3}=\alpha_{i} \cdot H, i=0,1,3,4$, of the Cartan by introducing the mass parameters $M_{i}$ as follows:

$$
M_{0}=-\frac{m_{1}+m_{2}}{2}, \quad M_{1}=\frac{m_{1}-m_{2}}{2}, \quad M_{3}=\frac{m_{3}-m_{4}}{2}, \quad M_{2}=\frac{m_{3}+m_{4}}{2} .
$$

The $N_{f}=4$ theory also exhibits a strong/weak coupling duality. The duality group acts via $\mathrm{SL}(2, \mathbb{Z})$ on the complexified gauge coupling $\tau$, which can be identified with the argument of the half-periods $e_{i}$ in the definition of the original Seiberg-Witten curve [6]. Simultaneously, it acts on the spectrum of the theory by permuting fundamental representations of the flavor symmetry $\mathrm{SO}(8)$ [6]. The full duality group of the theory is hence $W\left(D_{4}\right) \rtimes \mathrm{SL}(2, \mathbb{Z})$. The subgroup $\Gamma(2) \subset \mathrm{SL}(2, \mathbb{Z})$ is a symmetry of the theory acting solely on the parameter $\tau$. Replacing $\tau$ by the parameter $x=\frac{e_{3}-e_{2}}{e_{1}-e_{2}}(\tau)$ makes this invariance manifest. The remaining symmetry group $W\left(D_{4}\right) \rtimes S_{3}$ is isomorphic to the Weyl group of the exceptional group $F_{4}$. Under $S_{3} \cong \mathrm{SL}(2, \mathbb{Z}) / \Gamma(2)$, the ratio $x$ transforms as a cross ratio and the representations of $\mathrm{SO}(8)$ are exchanged by triality. The precise implementation of the semi-direct product $W\left(D_{4}\right) \rtimes \mathrm{SL}(2, \mathbb{Z})$ on the coupling and the Lie algebra is reviewed in appendix $\mathrm{C}$. 
The mass parameters $m_{i}$ (or $M_{i}$ ) can be organized in terms of the $\mathrm{SO}(8)$ invariants

$$
\begin{aligned}
R & =\frac{1}{2} \sum_{i} m_{i}^{2}, \\
T_{1} & =\frac{1}{12} \sum_{i<j} m_{i}^{2} m_{j}^{2}-\frac{1}{24} \sum_{i} m_{i}^{4}, \\
T_{2} & =-\frac{1}{24} \sum_{i<j} m_{i}^{2} m_{j}^{2}+\frac{1}{48} \sum_{i} m_{i}^{4}-\frac{1}{2} \prod_{i} m_{i}, \\
T_{3} & =-\frac{1}{24} \sum_{i<j} m_{i}^{2} m_{j}^{2}+\frac{1}{48} \sum_{i} m_{i}^{4}+\frac{1}{2} \prod_{i} m_{i}, \\
N & =\frac{3}{16} \sum_{i<j<k} m_{i}^{2} m_{j}^{2} m_{k}^{2}-\frac{1}{96} \sum_{i \neq j} m_{i}^{2} m_{j}^{4}+\frac{1}{96} \sum_{i} m_{i}^{6},
\end{aligned}
$$

such that $R$ and $N$ are invariant under triality, while the $T_{i}$ are permuted [6].

\subsection{The parameter map}

The mapping of parameters that identifies the four-point conformal block $\mathcal{F}_{14}^{23}$ of a conformal field theory with central charge $c$, insertions of conformal dimension $h_{i}$ and exchanged dimension $h$ with the instanton partition function $Z_{\text {inst }}$ of the gauge theory with deformation parameters $\epsilon_{i}$, masses $M_{i}$ and vector adjoint scalar vacuum expectation value $a$ is [1]

$$
c=1+6 Q^{2}, \quad Q=b+b^{-1}, \quad b=\sqrt{\frac{\epsilon_{2}}{\epsilon_{1}}}, \quad h_{i}=\frac{Q^{2}}{4}-\frac{M_{i}^{2}}{\epsilon_{1} \epsilon_{2}}, \quad h=\frac{Q^{2}}{4}-\frac{a^{2}}{\epsilon_{1} \epsilon_{2}},
$$

or equivalently, in terms of the exponents $\alpha_{i}$ of Liouville vertex operators,

$$
\alpha_{i}=\frac{Q}{2}-\frac{M_{i}}{\sqrt{\epsilon_{1} \epsilon_{2}}}, \quad \alpha=\frac{Q}{2}-\frac{a}{\sqrt{\epsilon_{1} \epsilon_{2}}} .
$$

Note that the massless limit $M_{i}=0$ corresponds to the insertion of four puncture operators of weight $\frac{Q^{2}}{4}$.

The U(1) correction factor discussed in [1] contributes $a$ independent terms to the leading terms $F^{(n, g)}, n+g \leq 1$, of the genus expansion of the partition function that we will analyze at length. These contributions will not be relevant to our discussion.

\section{The null vector decoupling equation}

To compute the four-point conformal block, we wish to pursue the same strategy we employed in [4] in the case of the one-point toroidal block: the correlator with a fifth insertion, chosen to be degenerate, satisfies a null vector decoupling equation. In fact, each individual conformal block contributing to the correlator satisfies the equation. Imposing appropriate monodromy conditions selects the solution coinciding with the exchange of a given momentum. In the semi-classical limit, we can extract the semi-classical four-point conformal block from this result. 
The five-point function with one degenerate insertion itself has a conjectured gauge theory interpretation. Indeed, the paper [10] proposes to identify the corresponding fivepoint conformal block with the instanton partition function in the gauge theory in the presence of a surface operator. According to this proposal, the two integers which label degenerate operators, see (3.1), determine the location of the surface operator in $\mathbb{R}^{4}$, while the position of the degenerate operator on the Riemann surface maps to two real parameters determining the type of surface operator.

It is hence worthwhile to discuss the null vector decoupling equation the five-point correlator satisfies in generality in this section, before solving it in the semi-classical limit in the next. We will begin by reviewing how this equation has arisen in the mathematical physics literature as a quantization of the Painlevé VI equation. By matching conformal field theory conventions to those employed in [17], we can translate the results presented there on the symmetries of the quantum Painlevé equation into our variables. Aside from recovering the gauge theory symmetries discussed in section 2.2 , we will witness an $\epsilon$ dependent deformation of one of these symmetries, as well as an enhancement, in the case of the instanton partition function in the presence of a surface operator. Finally, we will review the mapping of the null vector decoupling equation into elliptic variables, the starting point for our semi-classical treatment in section 4 .

\subsection{The null vector decoupling equation in spherical coordinates}

We consider four primary vertex operators $V_{h_{i}}$ of weight $h_{i}$ in a conformal field theory on the sphere, or the infinite plane, inserted at points $z_{i}$. To this four-point correlator, we add a fifth, degenerate field, which is to stay light in the semi-classical limit $b \rightarrow 0$. The degenerate weight

$$
h_{m n}=\frac{Q^{2}}{4}-\frac{1}{4}\left(m b+n b^{-1}\right)^{2}
$$

of lowest degree which remains light in the semi-classical limit is carried by the primary field $V_{(2,1)}$ of conformal dimension $h_{2,1}=-\frac{1}{2}-\frac{3}{4} b^{2}$. Imposing decoupling of this Virasoro null vector leads to a second order differential equation on the five-point function,

$$
\left[\partial_{z}^{2}+b^{2}\left(\sum_{k=0}^{3} \frac{h_{k}}{\left(z-z_{k}\right)^{2}}+\frac{\partial_{k}}{z-z_{k}}\right)\right]\left\langle V_{(2,1)}(z) V_{h_{0}}\left(z_{0}\right) \ldots V_{h_{3}}\left(z_{3}\right)\right\rangle=0 .
$$

Mapping the points $\left(z_{0}, z_{1}, z_{2}, z_{3}\right)$ to $(\infty, 1, x, 0)$, the null vector decoupling equation on the five-point correlator assumes the form

$$
\begin{aligned}
0= & {\left[\frac{1}{b^{2}} \partial_{z}^{2}+\frac{2 h_{0}}{z(z-1)}+\frac{h_{1}}{(z-1)^{2}}+\frac{h_{2}}{(z-x)^{2}}+\frac{h_{3}}{z^{2}}\right.} \\
& -\frac{1}{z(z-1)(z-x)}\left[(z-x) \sum_{i=0}^{3} h_{i}-(x-1) x \partial_{x}\right] \\
& \left.+\frac{1}{z(z-1)}\left[-h_{2,1}+(1-2 z) \partial_{z}\right]\right] \Psi_{5}(z, x) .
\end{aligned}
$$


with

$$
\Psi_{5}(z, x)=\left\langle V_{h_{0}}(\infty) V_{(2,1)}(z) V_{h_{1}}(1) V_{h_{2}}(x) V_{h_{3}}(0)\right\rangle .
$$

\subsection{Symmetries of the null vector decoupling equation}

In the present subsection, we will relate the null vector decoupling equation (3.3) to the quantized Painlevé VI equation. This will allow us to exploit the results of [17] to elucidate the symmetry group of the null vector decoupling equation and the transformation properties of the five-point correlator in the two-dimensional theory. The correspondence [10] then yields a prediction for the transformation of the vacuum expectation value of a surface operator in the $\epsilon$-deformed four-dimensional gauge theory under strong-weak coupling duality. The most interesting transformation rule is non-local, implemented via a Laplace transform. We stress that the discussion in this subsection is valid at finite $\epsilon_{1}$ and $\epsilon_{2}$.

We begin by reviewing how the null vector decoupling equation has arisen in the mathematics literature in the context of the theory of differential equations and their quantization. The study of (rational) second order ordinary differential equations which have solutions of which the movable singularities are necessarily poles [14], led to a classification of such equations [16]. The complete set contains six equations, referred to as the Painlevé equations I through VI. The Painlevé VI equation is the most general one from which all others can be obtained through degeneration limits. This equation also arises as the differential equation satisfied by the singularities of a second order Fuchsian equation with four regular singular points on the two-dimensional sphere, when one imposes the property that the monodromy of the solution be preserved [15]. The analysis of isomonodromic deformations $[19,20]$ of differential equations leads to a rich connection [21] with the theory of integrable systems (see e.g. [22]). The second order Painlevé differential equations can be re-expressed as a first order Hamiltonian system with interesting algebraic geometric implications [23]. More recently, the Hamiltonian Painlevé systems have been quantized [12], preserving the full symmetry of the classical problem [24]. The resulting Schrödinger equation is essentially the null vector decoupling equation (3.3). ${ }^{5}$

In [17], it was demonstrated that the full symmetry group of the Painlevé VI equation, determined to be the affine Weyl group $W\left(F_{4}^{(1)}\right)$ of the exceptional Lie algebra $F_{4}$ in [23], acts on the quantum equation via Bäcklund transformations: solutions to the equation can

\footnotetext{
${ }^{5}$ Concretely, the quantum Painlevé Schrödinger equation in the symmetrized form of equation (2.1) in [17] acting on a wave-function $\Psi_{\text {sym }}$ is equivalent to the null vector decoupling equation acting on our five-point correlator $\Psi_{5}$ on the sphere under the following identification of parameters (to avoid an internal clash of notation, the quantities $\beta_{i}$ refer to the quantities denoted $\alpha_{i}$ in [17] and it should be understood
} that $(q, z)$ in $[17]$ are denoted $(z, x)$ here):

$$
\beta_{1}=\frac{2 M_{0}}{\epsilon_{1}}, \quad \beta_{3}=\frac{2 M_{1}}{\epsilon_{1}}, \quad \beta_{0}=\frac{2 M_{2}}{\epsilon_{1}}, \quad \beta_{4}=\frac{2 M_{3}}{\epsilon_{1}}, \quad \beta_{2}=\frac{1}{2} \frac{\epsilon_{2}}{\epsilon_{1}}-\frac{\sum_{i=0}^{3} M_{i}}{\epsilon_{1}}, \quad \kappa=-b^{2},
$$

and

$$
\begin{aligned}
\Psi_{5}= & (1-x)^{-\frac{1}{6 b^{2}}-\frac{1}{2}-\frac{b^{2}}{4}+\frac{1}{\epsilon_{1} \epsilon_{2}}\left(M_{1}^{2}+M_{2}^{2}\right)} x^{-\frac{1}{6 b^{2}}-\frac{1}{2}-\frac{b^{2}}{4}+\frac{1}{\epsilon_{1} \epsilon_{2}}\left(M_{2}^{2}+M_{3}^{2}\right)} \\
& (x-z)^{\frac{1}{2}+\frac{b^{2}}{2}-\frac{M_{2}}{\epsilon_{1}}}(z-1)^{\frac{1}{2}+\frac{b^{2}}{2}-\frac{M_{1}}{\epsilon_{1}}} z^{\frac{1}{2}+\frac{b^{2}}{2}-\frac{M_{3}}{\epsilon_{1}}} \Psi_{\text {sym }} .
\end{aligned}
$$


be mapped to solutions to a transformed equation obtained by an action of $W\left(F_{4}^{(1)}\right)$ on the weights and the parameter $x$. By our discussion above, these results of [17] predict the transformation properties of the gauge theory instanton partition function in the presence of a surface operator under the action of this symmetry group.

We can compare the symmetry group $W\left(F_{4}^{(1)}\right)$ of quantum Painlevé, and hence of the five-point function, to the symmetry $W\left(D_{4}\right) \rtimes \mathrm{SL}(2, \mathbb{Z})$ of the $\epsilon$-undeformed gauge theory reviewed in section (2.2). The affine Weyl group of $F_{4}$ can be expressed as $W\left(F_{4}^{(1)}\right)=$ $W\left(D_{4}^{(1)}\right) \rtimes S_{4}$, with $\operatorname{Aut}_{D}\left(D_{4}^{(1)}\right)=S_{4}$ the group of automorphisms of the affine Dynkin diagram of $D_{4}^{(1)}[25]$. As the $\Gamma(2)$ factor in $\operatorname{SL}(2, \mathbb{Z})=\Gamma(2) \rtimes \mathrm{S}_{3}$ is invisible when the theory is expressed in terms of the cross ratio $x$, we recognize that the two symmetry groups differ by two generators, due to replacing the algebra $D_{4}$ by its affine counterpart $D_{4}^{(1)}$, and the group of Dynkin diagram automorphisms $\operatorname{Aut}_{D}\left(D_{4}\right)=S_{3}$ by its affine counterpart $\operatorname{Aut}_{D}\left(D_{4}^{(1)}\right)=S_{4}$. Considering the action of the symmetry generators on the weights and the variable $x$, we observe that the Weyl symmetry exchanging $m_{2}$ and $m_{3}$ of the gauge theory is deformed to the following symmetry

$$
\left(m_{1}, m_{2}, m_{3}, m_{4}\right) \rightarrow\left(m_{1}, m_{3}-\frac{\epsilon_{2}}{2}, m_{2}+\frac{\epsilon_{2}}{2}, m_{4}\right)
$$

of the five-point correlator. This deformation is also responsible for the occurrence of the two additional generators. In the $\epsilon_{2} \rightarrow 0$ limit, they cease to be independent. Note that the four point correlator retains the undeformed symmetry, as exemplified in section 4.3.

The transformation properties of the partition function under $W\left(F_{4}^{(1)}\right)$ mainly involve rescaling. The transformation (3.7) however induces Laplace transformations with regard to the insertion point of the degenerate operator, giving rise to a non-local transformation rule for the partition function. It would be very interesting to verify this transformation directly from the gauge theory perspective.

\subsection{The null vector decoupling equation in elliptic form}

The four-point conformal block depends on the cross ratio $x$ of the four insertion points. As discussed in section 2.1, the four insertion points can be mapped to the 2-torsion points (including the trivial one) of a torus, such that the modular parameter of the torus is related to the cross ratio $x$ via relation (2.10).

To study the null vector decoupling equation (3.3) of the five-point correlator, it proves advantageous to map this equation to elliptic variables, extending the map between the 2 -torsion points on the torus and the insertion point of the four-point function to a two-toone map between torus and sphere. This map was performed in $[5,14,26]$ and the result was identified $[11,27]$ as an elliptic integrable system of Inozemtsev type $B C_{1}[28,29]$. The latter in turn is equivalent to a Gaudin system [30] with four punctures after a reduction by an involution [31] (a relation that goes back to [15, 19]). For a history of the derivation, see e.g. [32]. The reference [13] provides further details for the long calculation, and the reference [33] states the full result in the conformal field theory context (for the heavy second order degenerate insertion). We provide many technical details of the derivation in our conventions and starting from the conformal field theory null vector decoupling equation in appendix A. Here we summarize the main features of the derivation. 
We introduce the coordinate $u$ on the torus via

$$
z=\frac{\wp(u)-e_{3}}{e_{1}-e_{3}} .
$$

The ansatz

$$
\Psi_{5}(z, x)=\frac{[(1-z) z(z-x)]^{\frac{1}{4}+\frac{b^{2}}{2}}}{[4 x(1-x)]^{\frac{1}{12}\left(1+3 b^{2}+8 h_{2}\right)}} \frac{\vartheta_{1}(u)^{b^{2}}}{\vartheta_{1}^{\prime}(0)^{\frac{1}{3}\left(1+b^{2}\right)}} \prod_{i=1}^{3}\left(\frac{\eta}{\vartheta_{i+1}(0)}\right)^{4 h_{i+2}} \psi(u, \tau)
$$

for the five-point correlator reduces the null vector decoupling equation (3.3) to a Schrödinger equation for $\psi(u, \tau)$,

$$
\left(\partial_{u}^{2}+4 b^{2} \sum_{i=0}^{3} \hat{h}_{i \wp}\left(u+\omega_{i}\right)\right) \psi(u, \tau)=-4 \pi i b^{2} \partial_{\tau} \psi(u, \tau) .
$$

Here, and in the following, we identify indices ${ }_{i} \bmod 4$. The symbols $\omega_{i}$ label the 2 -torsion points of the torus (with $\omega_{0}=0$ the trivial one). The parameters $\hat{h}_{i}$ are related to the conformal dimensions $h_{i}$ via a shift,

$$
\hat{h}_{i}=h_{i}-\frac{b^{2}}{4}-\frac{3}{16 b^{2}}-\frac{1}{2} .
$$

We refer to appendix A for the detailed derivation. We note that the weight dependent part of the prefactor in equation (3.9),

$$
\begin{aligned}
& {[4 x(1-x)]^{-\frac{2}{3} h_{2}} \prod_{i=1}^{3}\left(\frac{\eta}{\vartheta_{i+1}(0)}\right)^{4 h_{i+2}} } \\
= & 2^{-\frac{4}{3} \sum_{i=0}^{3} h_{i}}[x(1-x)]^{\frac{h_{0}}{3}}\left[\frac{x}{(1-x)^{2}}\right]^{\frac{h_{1}}{3}}[x(1-x)]^{-\frac{2}{3} h_{2}}\left[\frac{1-x}{x^{2}}\right]^{\frac{h_{3}}{3}},
\end{aligned}
$$

transforms as the four-point correlator under channel duality.

\subsection{The monodromy}

We will be searching for very particular solutions to the elliptic differential equation. To solve for the four-point conformal block, we need to impose that the momentum exchanged between the insertions at 0 and $x$ has a given value $a$, before the exchanged excitation bifurcates again into the states inserted at 1 and $\infty$. To avoid excess notation, we will indicate that this projection on to intermediate momentum $a$ has taken place simply by including the argument in the notation. Hence $\Psi_{4}$ will indicate the correlator, $\Psi_{4}(a)$ the corresponding conformal block.

We consider the operator product expansion of $V_{h_{3}}(0)$ and $V_{h_{2}}(x)$, yielding operators $V_{\alpha}(x)$, and then the operator product expansion of the degenerate insertion with each of these operators:

$$
\begin{aligned}
V_{h_{21}}(z) V_{\alpha}(x)= & C_{21, h_{\alpha}}^{h_{\alpha_{+}}}(z-x)^{h_{\alpha_{+}}-h_{21}-h_{\alpha}} V_{\alpha_{+}}(x)+\ldots \\
& +C_{21, h_{\alpha}}^{h_{\alpha_{-}}}(z-x)^{h_{\alpha_{-}}-h_{21}-h_{\alpha}} V_{\alpha_{-}}(x)+\ldots
\end{aligned}
$$


with $\alpha_{ \pm}=\alpha \pm \frac{b}{2}$. The monodromy which arises when the degenerate insertion at $z$ circles the operators at $x$ and 0 is determined by the exponent

$$
h_{\alpha_{ \pm}}-h_{21}-h_{\alpha}=\frac{b^{2}}{2} \pm \frac{a b}{\sqrt{\epsilon_{1} \epsilon_{2}}}+\frac{1}{2},
$$

where we have set $h_{\alpha}=\frac{Q^{2}}{4}-\frac{a^{2}}{\epsilon_{1} \epsilon_{2}}$.

In terms of the elliptic variable $u$ introduced in (3.8) of periods $\omega, \omega^{\prime}$, encircling the points 0 and $x$ on the sphere in anti-clockwise direction maps to traversing the cycle $\left[\kappa \omega^{\prime}, \kappa \omega^{\prime}+\omega\right]$ with $0<\kappa<\frac{1}{2}$. The prefactor in (3.9) relating $\Psi_{5}$ to $\psi$ induces a monodromy $\exp \left[\pi i\left(b^{2}+1\right)\right]$. The monodromy we must impose on the function $\psi(u, \tau)$ is hence

$$
\exp \left[ \pm \frac{2 \pi i a b}{\sqrt{\epsilon_{1} \epsilon_{2}}}\right]
$$

Note that a five-point conformal block should ordinarily depend on two intermediate weights. When one insertion is degenerate, the constrained operator product expansion (3.12) replaces one such dependence by a discrete label, reflected in the choice of sign in (3.14).

\section{The genus expansion}

To make contact with the topological string partition function in the field theory limit and the quasi-modular behavior of the conformal block that it predicts, we would like to compute the four-point spherical block in an expansion in the topological string coupling $g_{s}$ and the deformation parameter $s$, related to the $\epsilon$-parameters via

$$
g_{s}^{2}=\epsilon_{1} \epsilon_{2}, \quad s=\left(\epsilon_{1}+\epsilon_{2}\right)^{2} .
$$

After reviewing the structure of quasi-modular forms for the group $\Gamma(2)$ of interest, we will discuss the appropriate limit in the conformal field theory to implement the genus expansion. We will then review a recursion relation [26] which permits the computation of the conformal block in this limit, thus yielding the corresponding topological string partition function, order by order in $q_{2}$ and to all orders in the parameters $g_{s}$ and $s$. By expanding in $g_{s}$ and $s$ and imposing quasi-modularity, we can determine all order results in $q_{2}$ after a finite number of recursion steps. As a proof of principle, we compute $F^{(n, g)}$, $n+g=2$, in this manner.

In section 4.4, we turn to the solution of the null vector decoupling equation (3.10) in the genus expansion limit. Extracting the four-point block from this computation requires taking the strict $\epsilon_{2} \rightarrow 0$ limit, yielding modular results order by order in the deformation parameter $s$ and at leading order in the topological string coupling $g_{s}$, and to all orders in the modular parameter $q_{2}$.

\subsection{Quasi-modular forms of $\Gamma(2)$}

The invariance of the $N_{f}=4$ theory would naively suggest that the $\tau$-dependence of all observables holomorphic in $\tau$ should be captured in terms of modular forms of $\Gamma(2)$. These 
form the space $M(\Gamma(2))$, which is spanned by the powers of theta-functions $\vartheta_{2}^{4}$ and $\vartheta_{3}^{4}$ of weight 2 , or equivalently, by the half-periods $e_{1}$ and $e_{2}$. Via the relation to topological strings, we know that quantities naively holomorphic in $\tau$ may in fact exhibit $\bar{\tau}$ dependence. A minimal extension of $M(\Gamma(2))$ to allow for this is the space of almost holomorphic modular forms $\widehat{M}(\Gamma(2))$. The elements of this space are polynomials in $1 / \operatorname{Im}(\tau)$, with coefficients holomorphic in $\tau$, which transform modularly under $\Gamma(2)$ (see e.g. [34]). The space $\widehat{M}(\Gamma(2))$ is obtained from $M(\Gamma(2))$ by adding the non-holomorphic Eisenstein series $\hat{E}_{2}$ as a further generator. A quasi-modular form of weight $k$ is the constant term in the $1 / \operatorname{Im}(\tau)$ expansion of an almost holomorphic modular form of weight $k$. The rings of almost holomorphic modular forms $\widehat{M}(\Gamma(2))$ and of quasi-modular forms $\widetilde{M}(\Gamma(2))$ are isomorphic, the isomorphism given by mapping the generator $\hat{E}_{2}$ to the holomorphic (non-modular) Eisenstein series $E_{2}$. The space of quasi-modular forms $\widetilde{M}(\Gamma(2))$ is closed under differentiation. Quasi-modular forms of $\Gamma(2)$ are naturally expanded in terms of the modular variable $q_{2}$, where $q_{n}=\exp \frac{2 \pi i \tau}{n}$.

By imposing quasi-modularity, we will be able to extract all order results in $q_{2}$ from the elliptic recursion relation reviewed in section 4.3. In our computations of the fourpoint spherical block in section 4.4, quasi-modularity will be manifest for the derivative of coefficients of the logarithm of the semi-classical conformal block (via evaluation of period integrals of the Weierstrass function $\wp$ ) in a genus expansion. We will then verify experimentally that these results also integrate to quasi-modular forms.

\subsection{The genus expansion limit}

The refined topological string partition function is defined as the following generating function of the topological string amplitudes:

$$
Z_{\text {top }}=\exp \sum_{n, g=0}^{\infty} F^{(n, g)} s^{n} g_{s}^{2 g-2} .
$$

It is natural to consider the $\epsilon_{1}, \epsilon_{2} \rightarrow 0$ limit in the conformal field theory to reproduce this expansion. This amounts via the $2 \mathrm{~d} / 4 \mathrm{~d}$ dictionary $(2.16)$ to the condition that the exchanged conformal dimension $h$ be much bigger than the central charge $c, h \gg c$. The leading behavior of the partition function (4.2) in this limit is reminiscent of the semiclassical limit $b \rightarrow 0$ of the conformal block. We know, through an analysis of the null vector decoupling equation, that the leading term in the semi-classical conformal block is of order $\exp \left(a^{2} / \epsilon_{1} \epsilon_{2}\right)$ in this limit [5]. Thus, we expect a semi-classical four-point spherical block of the form

$$
\Psi_{4}(a)=\exp \left[\frac{1}{\epsilon_{1} \epsilon_{2}} F\left(\epsilon_{1}, \epsilon_{2}\right)\right]
$$

with $F$ a formal power series in $\epsilon_{1}$ and $\epsilon_{2}$. Note that equating this block with (4.2) predicts constraints on the terms in the power series $F$. Aside from the symmetry between $\epsilon_{1}$ and $\epsilon_{2}$ that is naively broken by the semi-classical limit, the absence of half-integer powers of the deformation parameter $s$ is non-trivial. Indeed, to reproduce this integral expansion from the $\epsilon$-deformed partition function of [18] requires an $\epsilon$-dependent shift of 
the mass parameters of [18]. The integral expansion arises naturally in the context of the holomorphic anomaly equations of topological string theory [35], and can be reproduced in conformal field theory both from the elliptic recursion relations and the null vector decoupling equations, as we discuss in this section.

\subsection{The elliptic recursion relation}

Conformal blocks are functions of the central charge $c$ of the theory, as well as of the exchanged weight $h$. Considered as functions of the central charge $c$ for fixed $h$, they exhibit poles at those values $c_{m n}$ of the central charge $c$ at which $h$ corresponds to a degenerate weight of degree $m n$. Considered as functions of the exchanged dimension $h$ for fixed $c$, they exhibit poles at degenerate weights $h_{m n}(c)$. In both cases [36], the residue of the poles is proportional to the conformal block itself, evaluated at the respective central charge or singular weight.

For the four-point conformal block on the sphere, making the poles in the central charge $c$ explicit gives rise to the formula

$$
F\left(c, h, h_{i}, x\right)=f\left(h, h_{i}, x\right)+\sum_{m, n} \frac{R_{m n}^{\prime}\left(h, h_{i}\right)}{c-c_{m n}(h)} F\left(c_{m n}, h+m n, h_{i}, x\right),
$$

where the function $R_{m n}^{\prime}\left(h, h_{i}\right)$ grows polynomially with $h$. The convergence of this expansion hence requires $c \gg h$. The function $f\left(h, h_{i}, x\right)$ is the infinite central charge limit of the conformal block. It can be determined in terms of hypergeometric functions to be

$$
f\left(h, h_{i}, x\right)=x^{h-h_{2}-h_{3}}{ }_{2} F_{1}\left(h+h_{2}-h_{3}, h+h_{0}-h_{1}, 2 h, x\right) .
$$

Due to the factor $x^{h}$ in the leading behavior and the shift $h \rightarrow h+m n$ on the right-hand side of (4.4), this equation gives rise to a recursion relation to determine the conformal block order by order in the cross ratio $x$ [37]. By the $2 \mathrm{~d} / 4 \mathrm{~d}$ dictionary, this yields the instanton expansion on the gauge theory side of the correspondence. In particular, the $\epsilon$-deformed partition function [18] yields a closed form solution to this recursion relation.

The genus expansion emerges from the second point of view. It proves convenient to write the conformal block as a product of functions of the elliptic parameter $q_{2}$,

$$
F\left(c, h, h_{i}, q_{2}\right)=f\left(c, h, h_{i}, q_{2}\right) H_{h}\left(c, h_{i}, q_{2}\right) .
$$

The rationale behind replacing the cross ratio $x$ dependence by $q_{2}$ dependence, with $q_{2}=$ $e^{\pi i \tau}$ and $\tau$ and $x$ related via (2.10), will become clear momentarily.

The first factor $f\left(c, h, h_{i}, q_{2}\right)$ yields the leading behavior of the conformal block in the $h \gg c \gg 1$ limit. It is computed in [5, 26] and given by

$$
\begin{aligned}
f\left(c, h, h_{i}, q_{2}\right) & =\left(16 q_{2}\right)^{h-\frac{c-1}{24}} x^{\frac{c-1}{24}-h_{2}-h_{3}}(1-x)^{\frac{c-1}{24}-h_{2}-h_{1}} \vartheta_{3}(0)^{\frac{c-1}{2}-4\left(h_{0}+h_{1}+h_{2}+h_{3}\right)}(4.7 \\
& =\left(16 q_{2}\right)^{-\frac{a^{2}}{\epsilon_{1} \epsilon_{2}}}\left(\frac{\vartheta_{3}^{2}}{\vartheta_{0} \vartheta_{2} \vartheta_{3}}\right)^{4 h_{2}}\left(\vartheta_{0} \vartheta_{2} \vartheta_{3}\right)^{Q^{2}} \prod_{i=1}^{3}\left(\vartheta_{i+1}\right)^{-4 h_{i+2}}
\end{aligned}
$$


The second factor in the block (4.6) satisfies the relation

$$
H_{h}\left(c, h_{i}, q_{2}\right)=1+\sum_{m, n}\left(16 q_{2}\right)^{m n} \frac{R_{m n}\left(c, h_{i}\right)}{h-h_{m n}(c)} H_{h_{m n}+m n}\left(c, h_{i}, q_{2}\right)
$$

with the residue factor given by $R_{m n}\left(c, h_{i}\right)=A_{m n} \prod_{i=1}^{4} Y_{r s}\left(2 m_{i}\right)$, and the functions $A$ and $Y$ by

$A_{m n}=\frac{1}{2}\left(\prod_{r=1}^{m} \prod_{-m}^{n}\right)^{\prime} \frac{1}{r b+s b^{-1}}, \quad Y_{m n}(M)=\left(\prod_{r=1-m}^{m-1} \prod_{s=1-n}^{n-1}\right)^{\prime \prime}\left(\frac{M}{\sqrt{\epsilon_{1} \epsilon_{2}}}-\frac{r b+s b^{-1}}{2}\right)$.

The prime on the first product indicates that the factors $(r, s)=(0,0),(m, n)$ are to be omitted, and the double prime on the second product prescribes a product over pairs satisfying $(r, s)=(1-m, 1-n) \bmod (2,2)$. The conformal dimensions $h_{m n}$ of degenerate representations are given in equation (3.1). The leading behavior $q_{2}^{h}$ of the conformal block [5] gives rise to a recursion relation with regard to the order in the elliptic parameter $q_{2}$, providing the rationale for introducing the elliptic parameter [26].

We will now exploit the elliptic recursion relation in various ways. Firstly, we note that the existence of the recursion relation allows us to rule out the convergence of the genus expansion (4.2) of the conformal block. This is most simply stated in the massless limit. In this limit, the partition function (4.2) takes the form

$$
\log Z_{\mathrm{top}}=\frac{1}{\epsilon_{1} \epsilon_{2}} \sum_{r, s}\left(\sum_{n} \psi_{n}^{r s} q_{2}^{n}\right) \epsilon_{1}^{r} \epsilon_{2}^{s}
$$

The coefficients $F^{(n, g)}\left(q_{2}\right)=\sum_{n} \psi_{n}^{r s} q_{2}^{n}$ sum to quasi-modular forms. The sum over $q_{2}$ is hence convergent in the upper half-plane. If the double sum (4.11) were convergent, hence absolutely convergent within the radius of convergence, we would be justified in reversing the order of summation. This is the order in which the recursion relation yields the conformal block, with

$$
\sum_{r s} \psi_{n}^{r s} \epsilon_{1}^{r} \epsilon_{2}^{s}=\frac{p_{n}\left(\epsilon_{1}, \epsilon_{2}\right)}{q_{n}\left(\epsilon_{1}, \epsilon_{2}\right)}
$$

a rational function in the $\epsilon$-parameters. As one easily verifies from the explicit expressions (4.10), the $\epsilon$-parameters in the function $q_{n}\left(\epsilon_{1}, \epsilon_{2}\right)$ are multiplied by integers of magnitude increasing with $n$. It follows that the radius of convergence of the series in $\epsilon_{1}$ and $\epsilon_{2}$ on the left-hand side of equation (4.12) decreases as $n$ grows. For any choice of the $\epsilon$-parameters, it ceases to converge for sufficiently large $n$. We conclude that the double sum (4.11) cannot be convergent.

The restriction to the massless case was not essential to this argument. In the massive case, the sum in parentheses in (4.11) is replaced by a more intricate expression. Via the holomorphic anomaly equation, it can be expressed in closed form as a quasi-modular form in a modular variable different from $q_{2}$ (namely the complex structure of the corresponding massive Seiberg-Witten curve), with coefficients depending on all parameters of the theory. Alternatively, it can be expressed as infinite sums over the mass parameters, 
with coefficients quasi-modular in $q_{2}$. Either way, the argument yielding non-convergence still holds.

As a second application of the elliptic recursion relation (4.9), we note that it yields a purely conformal field theoretic method of determining the topological string amplitudes $F^{(n, g)}$ in a mass expansion, with coefficients that are quasi-modular forms of $\Gamma(2)$, the subgroup of the S-duality group that does not act on the masses. At order $m$ in the masses, the weight of the quasi-modular coefficient is $w=2(n+g-1)+m$. As the space of quasimodular forms of this weight is a vector space of dimension $\left(\begin{array}{c}\frac{w}{2}+2 \\ 2\end{array}\right)$, the exact expression for the partition sum $F^{(n, g)}$ at a given order in the masses can be determined from this number of coefficients in $q_{2}$, hence after a finite number of recursion steps. It follows from (4.10) that the maximal order in the mass parameters is bounded at any given order in $q_{2}$. Hence, the quasi-modular coefficients of terms of high order in mass must conspire such that low orders in the modular parameter cancel. Analogous considerations apply to the case of the toroidal one-point block discussed in [4].

To demonstrate the utility of this approach in practice, we determined the partition sums $F^{(n, g)}$ for $n+g=2$, to order $a^{-6}$ in the exchanged conformal dimension from the elliptic recursion relation. The results are

$$
\begin{aligned}
F^{(0,2)}= & \frac{1}{32 a^{2}} E_{2}+\frac{1}{2880 a^{4}}\left(95 E_{2}^{2}+49 E_{4}\right) R+\frac{1}{a^{6}}\left(\left(\frac{145}{5184} E_{2}^{3}+\frac{169}{4320} E_{2} E_{4}+\frac{421}{25920} E_{6}\right) R^{2}\right. \\
& \left.+\left(-\frac{11}{64 \pi^{2}} E_{2}^{2}-\frac{31}{192 \pi^{2}} E_{4}\right)\left(e_{1} T_{1}+e_{2} T_{2}+e_{3} T_{3}\right)-\frac{1}{2 \pi^{4}} E_{2}\left(e_{1}^{2} T_{1}+e_{2}^{2} T_{2}+e_{3}^{2} T_{3}\right)\right), \\
F^{(1,1)}= & -\frac{1}{24 a^{2}} E_{2}-\frac{1}{360 a^{4}}\left(10 E_{2}^{2}+11 E_{4}\right) R+\frac{1}{a^{6}}\left(\left(-\frac{1}{54} E_{2}^{3}-\frac{11}{240} E_{2} E_{4}-\frac{71}{2160} E_{6}\right) R^{2}\right. \\
& \left.+\left(\frac{11}{144 \pi^{2}} E_{2}^{2}+\frac{5}{16 \pi^{2}} E_{4}\right)\left(e_{1} T_{1}+e_{2} T_{2}+e_{3} T_{3}\right)+\frac{7}{12 \pi^{4}} E_{2}\left(e_{1}^{2} T_{1}+e_{2}^{2} T_{2}+e_{3}^{2} T_{3}\right)\right), \\
F^{(2,0)}= & \frac{1}{96 a^{2}} E_{2}+\frac{1}{960 a^{4}}\left(5 E_{2}^{2}+9 E_{4}\right) R+\frac{1}{a^{6}}\left(\left(\frac{5}{1728} E_{2}^{3}+\frac{7}{720} E_{2} E_{4}+\frac{101}{8640} E_{6}\right) R^{2}\right. \\
& \left.+\left(-\frac{5}{576} \pi^{2} E_{2}^{2}-\frac{7}{64 \pi^{2}} E_{4}\right)\left(e_{1} T_{1}+e_{2} T_{2}+e_{3} T_{3}\right)-\frac{1}{12 \pi^{4}} E_{2}\left(e_{1}^{2} T_{1}+e_{2}^{2} T_{2}+e_{3}^{2} T_{3}\right)\right) .
\end{aligned}
$$

Up to order $a^{-4}$, these results match the results of [38] determined using the recursion relation in the cross ratio $x$. The term in $F^{(2,0)}$ of order $a^{-6}$ matches the result we will determine using the null vector decoupling equation.

\subsection{Solving the null vector decoupling equation in the genus expansion}

To solve the null vector decoupling equation (3.10) in the semi-classical limit, we use the following ansatz motivated by Liouville theory (as explained e.g. in [4]):

$$
\psi(u, \tau)=\exp \left[\frac{1}{\epsilon_{1} \epsilon_{2}} \mathcal{F}(\tau)+\frac{1}{\epsilon_{1}} \mathcal{W}(u, \tau)+O\left(\epsilon_{2}\right)\right] .
$$

With the dictionary (2.16), the differential equation in the $\epsilon_{2} \rightarrow 0$ limit becomes

$$
-\frac{1}{\epsilon_{1}} \mathcal{W}^{\prime \prime}(z \mid \tau)-\frac{1}{\epsilon_{1}^{2}} \mathcal{W}^{\prime}(z \mid \tau)^{2}+\sum_{i=0}^{3}\left(\frac{4}{\epsilon_{1}^{2}} M_{i}^{2}-\frac{1}{4}\right) \wp\left(z+\omega_{i}\right)=2(2 \pi i)^{2} \frac{1}{\epsilon_{1}^{2}} q \partial_{q} \mathcal{F}(\tau) .
$$


Note that only the derivative of $\mathcal{F}$ appears, as the normalization of $\psi(u, \tau)$ is neither fixed by the differential equation nor by the monodromy constraint.

Making a power series ansatz in $\epsilon_{1}$ for both $\mathcal{F}(\tau)$ and $\mathcal{W}(z \mid \tau)$,

$$
\mathcal{F}(\tau)=\sum_{n=0}^{\infty} \mathcal{F}_{n}(\tau) \epsilon_{1}^{n}, \quad \mathcal{W}(z \mid \tau)=\sum_{n=0}^{\infty} \mathcal{W}_{n}(z \mid \tau) \epsilon_{1}^{n},
$$

we obtain the system of equations

$$
\begin{aligned}
-\mathcal{W}_{0}^{\prime 2}+4 \sum_{i=0}^{3} M_{i}^{2} \wp\left(z+\omega_{i}\right) & =2(2 \pi i)^{2} q \partial_{q} \mathcal{F}_{0}, \\
-\mathcal{W}_{0}^{\prime \prime}-2 \mathcal{W}_{0}^{\prime} \mathcal{W}_{1}^{\prime} & =2(2 \pi i)^{2} q \partial_{q} \mathcal{F}_{1}, \\
-\mathcal{W}_{1}^{\prime \prime}-\mathcal{W}_{1}^{\prime 2}-2 \mathcal{W}_{0}^{\prime} \mathcal{W}_{2}^{\prime}-\frac{1}{4} \sum_{i=0}^{3} \wp\left(z+\omega_{i}\right) & =2(2 \pi i)^{2} q \partial_{q} \mathcal{F}_{2}, \\
-\mathcal{W}_{n}^{\prime \prime}-\sum_{i=0}^{n+1} \mathcal{W}_{i}^{\prime} \mathcal{W}_{n+1-i}^{\prime} & =2(2 \pi i)^{2} q \partial_{q} \mathcal{F}_{n+1} \quad \text { for } \quad n \geq 2 .
\end{aligned}
$$

The monodromy condition is imposed by demanding

$$
\oint \mathcal{W}_{0}^{\prime}= \pm 2 \pi i a, \quad \oint \mathcal{W}_{i}^{\prime}=0 \text { for } i>0
$$

The solution of the system of equations (4.16) - (4.19) proceeds much as in [4]. For the massless case, we can solve exactly in terms of quasi-modular forms in $\tau$. (Exceptions to non-quasi-modularity are the terms in $\mathcal{F}_{0}$ and $\mathcal{F}_{2}$ at order $a^{2}$ and $a^{0}$.) For the massive case, we define a second perturbation expansion in the parameters

$$
v_{i}=\left(\frac{M_{i}}{\pi a}\right)^{2}
$$

The contour integrals that one needs to perform to impose the monodromy conditions (4.20) generalize those of [4]. In particular, the integrands include products of the Weierstrass $\wp-$ function and their derivatives evaluated at arguments shifted by 2 -torsion points. We derive a set of recursion relations to calculate the monodromies of such integrands in appendix B. We can thus compute $\mathcal{F}(\tau)$, up to an integration constant, recursively order by order in $\epsilon_{1}$ and the $v_{i}$, and exactly at each such order in the modular parameter $q_{2}$.

Our solution algorithm hence gives rise to $q \partial_{q} \mathcal{F}_{n}$ as elements of the ring

$$
q \partial_{q} \mathcal{F}_{n} \in \mathbb{C}\left[\frac{1}{a}\right]\left[E_{2}, e_{1}, e_{2}\right]\left[\left[\frac{M_{i}}{a}\right]\right], \quad n>2 .
$$

In fact, more is true. As predicted by the $2 \mathrm{~d} / 4 \mathrm{~d}$ correspondence and analogously to the observations in the case of the torus one-point function in [4], $q \partial_{q} \mathcal{F}_{n}$ lies in the subring of derivatives of quasi-modular forms, such that $\mathcal{F}_{n}$ itself is an element of the right-hand side of (4.22). We have to date been able to verify this latter property merely experimentally. 
The statement that $q \partial_{q} \mathcal{F}_{n}$ integrates to a quasi-modular form is of course only true for an appropriate choice of integration constant. For ease of notation, we shall cite our results below with this choice implemented, which we indicate by a superscript 'c' (for 'complete'). Given the normalization (2.6), the proper choice of integration constant however is zero; the constant terms of the quasi-modular forms are provided by the product of Liouville three-point functions multiplying the conformal blocks in equation (2.6).

As anticipated in section 4.1, the subgroup $\Gamma(2)$ of the S-duality group that acts only on $\tau$ is realized by the fact that the $\tau$-dependence of our results is in terms of quasimodular forms of $\widetilde{M}(\Gamma(2))$. The full S-duality group $\mathrm{SL}(2, \mathbb{Z}) \rtimes \mathrm{W}\left(\mathrm{D}_{4}\right)$ is realized in an intricate manner already at play in [6]: the action of $\Gamma(2)$ on $e_{1}$ and $e_{2}$ can be extended to that of $\operatorname{SL}(2, \mathbb{Z})=\Gamma(2) \rtimes \mathrm{S}_{3}$ on $\left(e_{1}, e_{2}, e_{3}=-e_{1}-e_{2}\right)$, with $S_{3}$ permuting the entries of this vector. Expressing our results in terms of the $\mathrm{SO}(8)$ Casimirs (2.15) makes the full S-duality symmetry manifest.

The proof that the odd terms vanish, $q \partial_{q} \mathcal{F}_{2 n+1}=0$, proceeds as in [4]. The nonvanishing results we obtain, to order six in $\epsilon_{1}$, are the following: ${ }^{6}$

$$
\begin{aligned}
\mathcal{F}_{0}^{c}= & -a^{2} \log q_{2}+4 R \log \eta+\frac{1}{a^{2}}\left(\frac{E_{2} R^{2}}{6}-\frac{e_{1} T_{1}+e_{2} T_{2}+e_{3} T_{3}}{\pi^{2}}\right) \\
& +\frac{1}{a^{4}}\left[\frac{E_{4} N}{5}+\left(\frac{E_{2}^{2}}{36}+\frac{E_{4}}{180}\right) R^{3}-\frac{E_{2} R\left(e_{1} T_{1}+e_{2} T_{2}+e_{3} T_{3}\right)}{3 \pi^{2}}-\frac{R\left(e_{1}^{2} T_{1}+e_{2}^{2} T_{2}+e_{3}^{2} T_{3}\right)}{2 \pi^{4}}\right] \\
& +\frac{1}{a^{6}}\left[\frac{2}{15} E_{2} E_{4} N R+\frac{2 E_{6} N R}{35}+\frac{5 E_{2}^{3} R^{4}}{648}+\frac{1}{270} E_{2} E_{4} R^{4}+\frac{11 E_{6} R^{4}}{22680}\right. \\
& -\frac{5 E_{2}^{2} R^{2}\left(e_{1} T_{1}+e_{2} T_{2}+e_{3} T_{3}\right)}{36 \pi^{2}}-\frac{E_{4} R^{2}\left(e_{1} T_{1}+e_{2} T_{2}+e_{3} T_{3}\right)}{12 \pi^{2}}+\frac{E_{2}\left(e_{1} T_{1}+e_{2} T_{2}+e_{3} T_{3}\right)^{2}}{6 \pi^{4}} \\
& \left.-\frac{E_{2} R^{2}\left(e_{1}^{2} T_{1}+e_{2}^{2} T_{2}+e_{3}^{2} T_{3}\right)}{3 \pi^{4}}+\frac{4}{189} E_{6}\left(T_{1} T_{2}+T_{1} T_{3}+T_{2} T_{3}\right)+\frac{e_{1}^{3} T_{1}^{2}+e_{2}^{3} T_{2}^{2}+e_{3}^{3} T_{3}^{2}}{\pi^{6}}\right]
\end{aligned}
$$

+ higher order terms in the masses,

$$
\begin{aligned}
\mathcal{F}_{2}^{c}= & -\log \eta-\frac{E_{2} R}{12 a^{2}} \\
& +\frac{1}{a^{4}}\left[-\frac{1}{48}\left(E_{2}^{2}+E_{4}\right) R^{2}+\frac{E_{2}\left(e_{1} T_{1}+e_{2} T_{2}+e_{3} T_{3}\right)}{12 \pi^{2}}+\frac{e_{1}^{2} T_{1}+e_{2}^{2} T_{2}+e_{3}^{2} T_{3}}{2 \pi^{4}}\right] \\
& +\frac{1}{a^{6}}\left[-\left(\frac{1}{30} E_{2} E_{4}+\frac{2 E_{6}}{15}\right) N-\left(\frac{5 E_{2}^{3}}{648}+\frac{2}{135} E_{2} E_{4}+\frac{17 E_{6}}{3240}\right) R^{3}\right. \\
& \left.+\left(\frac{5 E_{2}^{2}}{72 \pi^{2}}+\frac{5 E_{4}}{24 \pi^{2}}\right) R\left(e_{1} T_{1}+e_{2} T_{2}+e_{3} T_{3}\right)+\frac{5 E_{2} R\left(e_{1}^{2} T_{1}+e_{2}^{2} T_{2}+e_{3}^{2} T_{3}\right)}{12 \pi^{4}}\right]
\end{aligned}
$$

+ higher order terms in the masses,

\footnotetext{
${ }^{6}$ We thank the authors of [38] for pointing out some typos in the following expressions in a previous version of this paper.
} 


$$
\begin{aligned}
\mathcal{F}_{4}^{c}= & \frac{E_{2}}{96 a^{2}}+\frac{1}{a^{4}}\left(\frac{E_{2}^{2}}{192}+\frac{3 E_{4}}{320}\right) R+\frac{1}{a^{6}}\left[\left(\frac{5 E_{2}^{3}}{1728}+\frac{7}{720} E_{2} E_{4}+\frac{101 E_{6}}{8640}\right) R^{2}\right. \\
& \left.-\left(\frac{5 E_{2}^{2}}{576 \pi^{2}}+\frac{7 E_{4}}{64 \pi^{2}}\right)\left(e_{1} T_{1}+e_{2} T_{2}+e_{3} T_{3}\right)-\frac{E_{2}}{12 \pi^{4}}\left(e_{1}^{2} T_{1}+e_{2}^{2} T_{2}+e_{3}^{2} T_{3}\right)\right] \\
& +\frac{1}{a^{8}}\left[\left(\frac{7 E_{2}^{2} E_{4}}{1440}+\frac{239 E_{4}^{2}}{1440}+\frac{E_{2} E_{6}}{30}\right) N+\left(\frac{35 E_{2}^{4}}{20736}+\frac{203 E_{2}^{2} E_{4}}{25920}+\frac{1193 E_{4}^{2}}{103680}+\frac{337 E_{2} E_{6}}{25920}\right) R^{3}\right. \\
& -\left(\frac{7 E_{2}^{3} R}{576 \pi^{2}}+\frac{161 E_{2} E_{4} R}{960 \pi^{2}}+\frac{77 E_{6} R}{480 \pi^{2}}\right)\left(e_{1} T_{1}+e_{2} T_{2}+e_{3} T_{3}\right) \\
& \left.-\left(\frac{7 E_{2}^{2}}{64 \pi^{4}}+\frac{77 E_{4}}{192 \pi^{4}}\right)\right] R\left(e_{1}^{2} T_{1}+e_{2}^{2} T_{2}+e_{3}^{2} T_{3}\right)+\text { higher order terms in the masses } \\
\mathcal{F}_{6}^{c}= & -\frac{1}{a^{4}}\left(\frac{E_{2}^{2}}{2304}+\frac{13 E_{4}}{11520}\right)-\frac{1}{a^{6}}\left(\frac{5 E_{2}^{3}}{10368}+\frac{E_{2} E_{4}}{432}+\frac{355 E_{6}}{72576}\right) R \\
& +\frac{1}{a^{8}}\left[\left(-\frac{35 E_{2}^{4}}{82944}-\frac{287 E_{2}^{2} E_{4}}{103680}-\frac{9235 E_{4}^{2}}{580608}-\frac{5671 E_{2} E_{6}}{725760}\right) R^{2}\right. \\
& +\left(\frac{7 E_{2}^{3}}{6912 \pi^{2}}+\frac{971 E_{2} E_{4}}{34560 \pi^{2}}+\frac{1267 E_{6}}{17280 \pi^{2}}\right)\left(T_{1} e_{1}+T_{2} e_{2}+T_{3} e_{3}\right) \\
& \left.+\left(\frac{7 E_{2}^{2}}{576 \pi^{4}}+\frac{95 E_{4}}{576 \pi^{4}}\right)\left(T_{1} e_{1}^{2}+T_{2} e_{2}^{2}+T_{3} e_{3}^{2}\right)\right] \\
& +\frac{1}{a^{10}}\left[-\left(\frac{E_{2}^{3} E_{4}}{1440}+\frac{89 E_{2} E_{4}^{2}}{1600}+\frac{1}{160} E_{2}^{2} E_{6}+\frac{4679 E_{4} E_{6}}{14400}\right) N\right. \\
& -\left(\frac{7 E_{2}^{5}}{20736}+\frac{7 E_{2}^{3} E_{4}}{2592}+\frac{22963 E_{2} E_{4}^{2}}{907200}+\frac{803 E_{2}^{2} E_{6}}{96768}+\frac{28963 R^{3} E_{4} E_{6}}{1036800}\right) R^{3} \\
& +\left(\frac{7 E_{2}^{4}}{3456 \pi^{2}}+\frac{125 E_{2}^{2} E_{4}}{2304 \pi^{2}}+\frac{20971 E_{4}^{2}}{48384 \pi^{2}}+\frac{1259 E_{2} E_{6}}{8064 \pi^{2}}\right) R\left(T_{1} e_{1}+T_{2} e_{2}+T_{3} e_{3}\right) \\
& \left.+\left(\frac{13 E_{2}^{3}}{576 \pi^{4}}+\frac{2077 E_{2} E_{4}}{5760 \pi^{4}}+\frac{3373 E_{6}}{5760 \pi^{4}}\right) R\left(T_{1} e_{1}^{2}+T_{2} e_{2}^{2}+T_{3} e_{3}^{2}\right)\right] \\
& \text { higher order terms in the masses. }
\end{aligned}
$$

The derivation of these results also required determining the quantities $\mathcal{W}_{n}^{\prime}(z)$ to particular orders in $\epsilon_{1}$ and the mass parameters.

The $2 \mathrm{~d} / 4 \mathrm{~d}$ correspondence implies the identification $F^{(n, 0)}=\mathcal{F}_{2 n}^{c}$. The vanishing of $\mathcal{F}_{2 n+1}$ is hence consistent with the fact that no half-integer powers of $s$ appear in the expansion (4.2). Our results in (4.23) confirm the massless results obtained via the holomorphic anomaly relations in [35], as well as the results in [39] and [38], of which the latter are based on combining instanton calculus with the demand for quasi-modularity, to the order given there. ${ }^{7}$ The paper [35] also gives closed results for the massive amplitudes, but as a function of both the UV coupling (the coupling $q_{2}$ considered here), and the effective coupling determined via special geometry. Furthermore, the results are expressed in terms of the Seiberg-Witten $u$ coordinate, rather than the flat coordinate $a$. To check against the

\footnotetext{
${ }^{7}$ There are minor typos in the signs in equation (3.22) in version 1 of [38].
} 
results of [35], we can generate our power series in masses by expressing the former purely in terms of $q_{2}$ and $a$.

The terms in the logarithm of the coefficient of $\psi(u, \tau)$ in (3.9) that scale as $\frac{1}{\epsilon_{2}}$ and thus contribute to $F^{(n, 0)}$ are

$$
-\frac{8}{3} h_{2} \log \frac{\vartheta_{0} \vartheta_{2}}{\vartheta_{3}^{2}}-4 \sum_{i=1}^{3} h_{i+2} \log \frac{\vartheta_{i+1}}{\eta}=8 h_{2} \log \frac{\vartheta_{3}}{\eta}-4 \sum_{i=1}^{3} h_{i+2} \log \frac{\vartheta_{i+1}}{\eta}+\text { const }
$$

where the last term is $\tau$-independent. These terms only contribute to $F^{(0,0)}$ and $F^{(1,0)}$, such that

$$
\begin{aligned}
F^{(0,0)} & =-8 M_{2}^{2} \log \frac{\vartheta_{3}}{\eta}+4 \sum_{i=1}^{3} M_{i+2}^{2} \log \frac{\vartheta_{i+1}}{\eta}+\mathcal{F}_{0}^{c} \\
& =-a^{2} \log q_{2}-4 M_{2}^{2} \log \frac{\vartheta_{3}^{2}}{\eta^{3}}+4 \sum_{i=1}^{3} M_{i+2}^{2} \log \vartheta_{i+1}+\text { quasi-modular forms }
\end{aligned}
$$

and

$$
F^{(1,0)}=\log \frac{\vartheta_{3}}{\vartheta_{0} \vartheta_{2}}+\text { quasi-modular forms }
$$

Note that we have thus reproduced the leading behavior (4.8) of the conformal block as determined in [5] to leading order in $\epsilon_{2}$.

\section{Conclusion}

In this paper, we continue our study of the duality between two-dimensional conformal field theory and four-dimensional $\mathcal{N}=2$ supersymmetric gauge theory [1] with an emphasis on the genus expansion. Following up on our analysis of the one-point toroidal block [4], we uncover modular properties of the spherical four-point conformal block. In particular, we show that when we assume quasi-modularity of the genus expansion coefficients, the elliptic recursion relations allow for their straightforward determination. At the same time, the recursion relation permits us to conclude that the genus expansion is formal, with vanishing radius of convergence. We demonstrate that in the study of the semi-classical limit of an appropriate null vector decoupling equation, quasi-modularity arises intrinsically. Upon making a power series ansatz for the five-point block, we obtain a system of equations for the expansion coefficients which can be studied on its own merits. The $\tau$-derivatives of these coefficients are manifestly quasi-modular. We have experimentally observed that they also integrate to quasi-modular forms.

The exact null vector decoupling equation on the five-point function is interesting in its own right, as it conjecturally governs the instanton partition function in the presence of an elementary surface operator [10]. By exploiting its relation to the quantum Painlevé VI equation [17], we have been able to determine the transformation properties of this gauge theory object under the action of the group $W\left(D_{4}^{(1)}\right) \rtimes \operatorname{Aut}_{D}\left(D_{4}^{(1)}\right)$, an enhancement of the symmetry group $W\left(D_{4}\right) \rtimes \operatorname{Aut}_{D}\left(D_{4}\right)$ for the $\epsilon$-undeformed $N_{f}=4$ gauge theory [6]. 
Our work gives rise to numerous questions worthy of further study. The predicted transformation properties of the instanton partition function in the presence of a surface operator need to be understood from the vantage point of gauge theory. In the same vein, the symmetry enhancement we observed needs to be investigated in the full $\epsilon$-deformed gauge theory. Furthermore, the identification of the null vector decoupling equation with the Inozemtsev integrable system remains to be fully exploited. Finally, quasi-modularity is the determining feature underlying our computations. It should be possible to identify a limit in parameter space of more general quiver gauge theories in which the introduction of elliptic or generalized modular variables yields similar structure. Proving quasi-modularity of the expansion coefficients determined via the null vector decoupling equation, finding an interpretation of this symmetry from within conformal field theory, and developing a better understanding of its occurrence against the backdrop of the non-convergence of the power series giving rise to it pose important open challenges.

\section{Acknowledgments}

We would like to thank Yuji Tachikawa for interesting correspondence. Our work is supported in part by the grant ANR-09-BLAN-0157-02.

\section{A The null vector decoupling equation in elliptic variables}

In this appendix, we explicitly perform the map between the null vector decoupling equation in spherical variables to the differential equation in elliptic variables. Our derivation combines results in references $[11,14,26,27,32,33]$; the details provided in [13] proved helpful.

\section{A.1 The change of variables}

The Weierstrass $\wp$-function defines a two-to-one map from the torus $\mathbb{C} / \Lambda$ to the sphere $\mathbb{C} \cup \infty$, mapping the 2-torsion points $\omega_{i}$ to the half-periods $e_{i}=\wp\left(\omega_{i}\right)$, and the origin to infinity. As we wish to map these points to the insertion points $(\infty, 1, x, 0)$ instead, we consider the map

$$
z=\frac{\wp(u)-e_{3}}{e_{1}-e_{3}}
$$

which in particular maps $\omega_{2}$ to $x$,

$$
x=\frac{e_{3}-e_{2}}{e_{3}-e_{1}}(\tau) .
$$

Note that in the following, $z$ will both specify a general coordinate on the sphere and the insertion point of the degenerate insertion, likewise for $u$ and the torus - we trust that this will not give rise to confusion. To invert the map (A.1) locally, note that the right-hand side is the inverse of the squared Jacobi sine function introduced in appendix A.2. We can hence invoke equation (A.26) to obtain

$$
u=\frac{1}{\sqrt{e_{1}-e_{3}}} \int_{0}^{\sqrt{\frac{1}{z}}} \frac{d y}{\sqrt{\left(1-y^{2}\right)\left(1-k^{2} y^{2}\right)}} .
$$


To convert the null vector decoupling equation

$$
\begin{array}{r}
{\left[-\frac{h_{(2,1)}}{(z-1) z}+\frac{h_{0}}{(z-1) z}+\frac{h_{1}}{(z-1)^{2} z}-\frac{\left(x^{2}+z-2 x z\right) h_{2}}{(x-z)^{2}(z-1) z}-\frac{h_{3}}{z^{2}(z-1)}\right.} \\
\left.-\frac{(x-1) x \partial_{x}}{(x-z)(z-1) z}+\frac{(1-2 z) \partial_{z}}{(z-1) z}+\frac{1}{b^{2}} \partial_{z}^{2}\right] \Psi_{5}(z, x)=0,
\end{array}
$$

where

$$
\Psi_{5}(z, x)=\left\langle V_{h_{0}}(\infty) V_{(2,1)}(z) V_{h_{1}}(1) V_{h_{2}}(x) V_{h_{3}}(0)\right\rangle,
$$

from spherical variables $(x, z)$ to elliptic variables $(\tau, u)$, we will need the following derivatives:

$$
\begin{aligned}
\frac{\partial u}{\partial z} & =\frac{1}{\wp^{\prime}} \frac{\partial \wp}{\partial z}=\frac{1}{2 \sqrt{e_{1}-e_{3}} \sqrt{z(z-1)(z-x)}}, \\
\frac{\partial \tau}{\partial x} & =\frac{4 \pi i}{\left(e_{1}-e_{3}\right) 4 x(x-1)}, \\
\frac{\partial u}{\partial x} & =\frac{\partial_{u} \log \vartheta_{0}(u)}{2\left(e_{1}-e_{3}\right)(1-x) x}-\frac{1}{2(x-1)} \sqrt{\frac{z-1}{\left(e_{1}-e_{3}\right) z(z-x)}} .
\end{aligned}
$$

The relations (A.6) and (A.7) are easily obtained from (A.21) and (A.36). To compute $\frac{\partial u}{\partial x}$, we have followed references $[13,27,32]$.

The ansatz

$$
\Psi_{5}(x, z)=x^{-\frac{b^{2}}{4}}((1-z) z(z-x))^{\frac{1}{4}+\frac{b^{2}}{2}} \vartheta_{1}(u)^{b^{2}} \psi(u, \tau)
$$

yields a differential equation for $\psi(u, \tau)$ with vanishing linear derivative in $u$. We have here used the identities (A.32), (A.35) and (A.36) to express

$$
\vartheta_{0}(u)=\frac{\vartheta_{1}(u)}{x^{\frac{1}{4}} \operatorname{sn}(2 K u)}=\frac{\sqrt{z}}{x^{\frac{1}{4}}} \vartheta_{1}(u),
$$

which renders the prefactor in (A.9) more symmetric.

To evaluate the $u$ - and $\tau$-derivatives on the prefactor of $\psi(u, \tau)$ in the definition (A.9), we need the derivative

$$
\begin{aligned}
\frac{\partial z}{\partial \tau} & =-\frac{\partial x}{\partial \tau} \frac{\partial u}{\partial x} / \frac{\partial u}{\partial z} \\
& =-\frac{1}{\pi}\left(i\left(e_{1}-e_{3}\right) x(z-1)+\partial_{u} \log \vartheta_{0} \sqrt{\left(e_{1}-e_{3}\right)} \sqrt{z(z-1)(z-x)}\right) .
\end{aligned}
$$

We can use the heat equation (A.30) and (A.32) to express $\partial_{u} \log \vartheta_{0}$ in terms of $x, z$ and $\eta$.

Upon dividing the equation (A.4) by $\left(\frac{\partial u}{\partial z}\right)^{2}$, analyzing the pole and vanishing structure of the terms proportional to the weights $h_{i}$ fixes the form of these terms in elliptic variables to be proportional to

$$
\sum_{i=0}^{3} h_{i}\left(\wp\left(u-\omega_{i}\right)-e_{i+2}\right)
$$


plus a $\tau$-dependent function. We have introduced $\omega_{0}=e_{0}=0$ and indexed the half-periods modulo 4 for ease of notation. The terms generated by our ansatz (A.9) conspire to shift the weights $h_{i}$ by a common term

$$
h_{i} \rightarrow h_{i}-\frac{b^{4}+2 b^{2}+\frac{3}{4}}{4 b^{2}}=: \hat{h}_{i} .
$$

After a detailed calculation, we arrive at the differential equation

$$
\left(\partial_{u}^{2}+4 b^{2} \sum_{i=0}^{3} \hat{h}_{i}\left(\wp\left(u+\omega_{i}\right)-e_{i+2}\right)\right) \psi=-\left(f(\tau)+4 \pi i b^{2} \partial_{\tau}\right) \psi
$$

with

$$
f(\tau)=\frac{b^{2}}{3}\left(\left(e_{3}-e_{1}\right)\left(1+8 h_{2}\right)-\left(e_{3}-e_{2}\right)\left(2+3 b^{2}+16 h_{2}\right)-6 \eta_{1}\left(1+b^{2}\right)\right) .
$$

As the function $f(\tau)$ is $u$-independent, it can be absorbed in a redefinition of the function $\psi$. Using the identities (A.36), we determine the required rescaling to be

$$
\psi(u, \tau) \rightarrow \vartheta_{1}^{\prime}(0)^{-\frac{1}{3}\left(1+b^{2}\right)} x^{-\frac{1}{12}\left(1+8 h_{2}\right)}(1-x)^{-\frac{1}{12}\left(1+3 b^{2}+8 h_{2}\right)} \psi(u, \tau) .
$$

Finally, we can also absorb the $u$-independent term

$$
\sum_{i=1}^{3} h_{i+2} e_{i}(\tau)
$$

in (A.14) via a shift

$$
\psi(u, \tau) \rightarrow \prod_{i=1}^{3}\left(\frac{\eta}{\vartheta_{i+1}(0)}\right)^{4 h_{i+2}} \psi(u, \tau) .
$$

We have here used the identity (A.34). The differential equation in its final form is thus given by

$$
\left(\partial_{u}^{2}+4 b^{2} \sum_{i=0}^{3} \hat{h}_{i \wp}\left(u+\omega_{i}\right)\right) \psi(u, \tau)=-4 \pi i b^{2} \partial_{\tau} \psi(u, \tau),
$$

where $\psi(u, \tau)$ is related to the five-point function (A.5) via the rescaling

$$
\Psi_{5}(z, x)=\frac{[(1-z) z(z-x)]^{\frac{1}{4}+\frac{b^{2}}{2}}}{[4 x(1-x)]^{\frac{1}{12}\left(1+3 b^{2}+8 h_{2}\right)}} \frac{\vartheta_{1}(u)^{b^{2}}}{\vartheta_{1}^{\prime}(0)^{\frac{1}{3}\left(1+b^{2}\right)}} \prod_{i=1}^{3}\left(\frac{\eta}{\vartheta_{i+1}(0)}\right)^{4 h_{i+2}} \psi(u, \tau) .
$$

We have chosen a overall normalization that is convenient in determining transformation properties under channel duality.

\section{A.2 Properties of elliptic and modular functions}

The Weierstrass $\wp$-function is an even function with a double pole at the origin. It satisfies the differential equation

$$
\wp^{\prime}(v)^{2}=4 \prod_{i=1}^{3}\left(\wp(v)-e_{i}\right)
$$


The $e_{i}$ are the half-periods of $\wp$. Since the function $\wp$ is even, we conclude that they are the images of the 2-torsion points of the torus under $\wp$,

$$
e_{i}=\wp\left(\omega_{i}\right) \text {. }
$$

Denoting the periods of $\wp$ as $\omega$ and $\omega^{\prime}$, we enumerate the 2-torsion points as $\omega_{1}=\frac{\omega}{2}$, $\omega_{2}=\frac{\omega+\omega^{\prime}}{2}, \omega_{3}=\frac{\omega^{\prime}}{2}$. With regard to a rescaling of its periods, the Weierstrass $\wp$-function behaves as

$$
\wp\left(v \mid \omega, \omega^{\prime}\right)=\frac{1}{\omega^{2}} \wp\left(\frac{v}{\omega} \mid 1, \frac{\omega^{\prime}}{\omega}\right) .
$$

When relating $\wp$ to theta functions below and in our computations in the body of this paper, we will normalize its periods to 1 and $\tau$ to simplify the formulae.

The Jacobi sine function provides a two-to-one odd map from the torus to the sphere. It relates to the Weierstrass function $\wp$ as follows,

$$
\operatorname{sn}^{2}\left(\sqrt{e_{1}-e_{3}} v\right)=\frac{e_{1}-e_{3}}{\wp(v)-e_{3}} .
$$

Defining $w=\sqrt{e_{1}-e_{3}} v$ and introducing the standard notation $K=\sqrt{e_{1}-e_{3}} \omega_{1}, i K^{\prime}=$ $\sqrt{e_{1}-e_{3}} \omega_{3}$, the sine function $\operatorname{sn}(w)$ has periodicities $4 K$ and $2 i K^{\prime}$, and moreover satisfies $\operatorname{sn}(w+2 K)=-\operatorname{sn}(w)$. The scaling of the argument of $\operatorname{sn}(w)$ in (A.24) is to render its periods independent of the choice of periods of $\wp$. The Jacobi sine function obeys the differential equation

$$
\operatorname{sn}^{\prime}(w)^{2}=\left(1-\operatorname{sn}(w)^{2}\right)\left(1-k^{2} \operatorname{sn}(w)^{2}\right)
$$

with $k^{2}=x$, and can hence be locally inverted via

$$
\operatorname{sn}^{-1}(z)=\int_{0}^{z} \frac{d y}{\sqrt{\left(1-y^{2}\right)\left(1-k^{2} y^{2}\right)}}
$$

The sine function with shifted argument satisfies the relations

$$
\begin{aligned}
\operatorname{sn}(w+K) & =\sqrt{\frac{1-\operatorname{sn}^{2}(w)}{1-k^{2} \operatorname{sn}^{2}(w)}}, \\
\operatorname{sn}\left(w+i K^{\prime}\right) & =\frac{1}{k \operatorname{sn}(w)}, \\
\operatorname{sn}\left(w+K+i K^{\prime}\right) & =\frac{1}{k \operatorname{sn}(w+K)} .
\end{aligned}
$$

These relations can be invoked to derive expressions for the shifted Weierstrass function $\wp\left(v+\omega_{i}\right)$ in terms of $\wp(v)$ and the half-periods.

Theta functions $\vartheta_{i}(u \mid \tau)=\vartheta_{i}(u), i=0, \ldots, 3$, are quasi-periodic functions associated to the lattice spanned by the lattice vectors 1 and $\tau$. Both the Weierstrass function $\wp$ and its half-periods can be expressed in terms of the theta functions $\vartheta_{i}$ and their $\tau$-derivatives, related to the $u$-derivative via the heat equation

$$
4 \pi i \partial_{\tau} \vartheta_{i}-\partial_{u}^{2} \vartheta_{i}=0
$$


Thus, the function $\wp$ can be expressed via the theta functions as

$$
\wp(u)=-\partial_{u} \log \vartheta_{1}-2 \eta_{1}
$$

and

$$
\sqrt{\wp(u)-e_{i}}=\frac{\vartheta_{1}^{\prime}(0)}{\vartheta_{i+1}(0)} \frac{\vartheta_{i+1}(u)}{\vartheta_{1}(u)},
$$

where indices are to be understood modulo 4 . We have here introduced the notation

$$
\eta_{1}=-\frac{2 \pi i}{3} \partial_{\tau} \log \vartheta_{1}^{\prime}(0)=-2 \pi i \partial_{\tau} \log \eta,
$$

with $\eta$ the Dedekind eta function that satisfies

$$
\eta=\left(\frac{\vartheta_{1}^{\prime}(0)}{2 \pi}\right)^{\frac{1}{3}} .
$$

Expanding the right-hand side of equation (A.32) in $u$ and recalling that the Weierstrass function expanded around the origin has no constant term yields the expressions

$$
e_{i}=-4 \pi i \partial_{\tau} \log \vartheta_{i+1}(0)-2 \eta_{1}
$$

for the half-periods in terms of theta functions. Evaluating equation (A.32) at the 2-torsion points and invoking

$$
\vartheta_{1}^{\prime}(u)=\pi \vartheta_{0}(u) \vartheta_{2}(u) \vartheta_{3}(u)
$$

gives rise respectively to the first equality in the following three identities:

$$
\begin{aligned}
& e_{1}-e_{2}=\pi^{2} \vartheta_{0}^{4}(0)=4 \pi i \partial_{\tau} \log \frac{\vartheta_{3}(0)}{\vartheta_{2}(0)}, \\
& e_{1}-e_{3}=\pi^{2} \vartheta_{3}^{4}(0)=4 \pi i \partial_{\tau} \log \frac{\vartheta_{0}(0)}{\vartheta_{2}(0)}, \\
& e_{2}-e_{3}=\pi^{2} \vartheta_{2}^{4}(0)=4 \pi i \partial_{\tau} \log \frac{\vartheta_{0}(0)}{\vartheta_{3}(0)} .
\end{aligned}
$$

The second then follow after recourse to equation (A.34).

\section{B The monodromies of shifted Weierstrass integrands}

In imposing the monodromy condition on our semi-classical conformal block, we need to compute the following types of integrals:

$$
K\left(n_{0}, n_{1}, n_{2}, n_{3}\right)=\oint_{\alpha} \wp(z)^{n_{0}} \wp\left(z+\omega_{1}\right)^{n_{1}} \wp\left(z+\omega_{2}\right)^{n_{2}} \wp\left(z+\omega_{3}\right)^{n_{3}} .
$$

This is a class of integrals generalizing those reviewed in [4] — we will freely use the results of that reference. In fact, we also encounter integrands containing an even number of derivatives of the Weierstrass function evaluated at half-periods, but these can be eliminated using the formulae

$$
\left(\wp^{\prime}\left(z+\omega_{i}\right)\right)^{2}=4 \wp\left(z+\omega_{i}\right)^{3}-g_{2} \wp\left(z+\omega_{i}\right)-g_{3}
$$




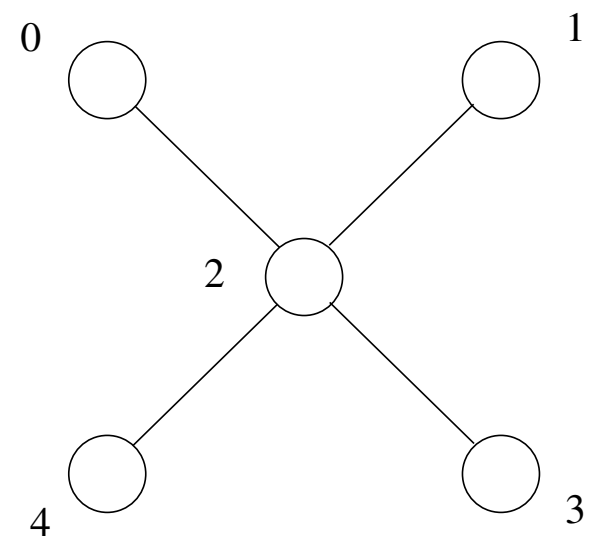

Figure 1. The Dynkin diagram of affine $D_{4}^{(1)}$.

and

$$
\begin{aligned}
\wp^{\prime}\left(z+\omega_{i}\right) \wp^{\prime}\left(z+\omega_{j}\right)= & 3\left(-\wp^{2}\left(z+\omega_{i}\right) \wp\left(z+\omega_{j}\right)-\wp\left(z+\omega_{i}\right) \wp^{2}\left(z+\omega_{j}\right)\right. \\
& +\frac{1}{12} g_{2}\left(\wp\left(z+\omega_{i}\right)+\wp\left(z+\omega_{j}\right)\right) \\
& \left.+e_{i j}\left(\wp\left(z+\omega_{i}\right)^{2}+\wp^{2}\left(z+\omega_{j}\right)-\frac{1}{6} g_{2}\right)\right)
\end{aligned}
$$

where $e_{i j}=\wp\left(\omega_{i}+\omega_{j}\right)$. Integrands with an odd number of derivatives vanish, as can be seen from the parity of the integrand and the symmetry of the integration domain. Finally, the integrals $K\left(n_{0}, n_{1}, n_{2}, n_{3}\right)$ can be computed using the identity

$$
\wp\left(z+\omega_{i}\right) \wp(z)=e_{i}\left(\wp\left(z+\omega_{i}\right)+\wp(z)\right)+e_{i}^{2}+\prod_{k \neq i} e_{k} .
$$

Indeed, by invoking this identity, the total order $n_{0}+n_{1}+n_{2}+n_{3}$ of the integrand of $K\left(n_{0}, n_{1}, n_{2}, n_{3}\right)$ can be lowered until the integrand is a power of a single shifted Weierstrass function, which can then be computed using the results of [4]. In other words, the recursion relation

$$
\begin{aligned}
K\left(n_{0}, n_{1}, n_{2}, n_{3}\right)= & e_{1}\left(K\left(n_{0}, n_{1}-1, n_{2}, n_{2}\right)+K\left(n_{0}-1, n_{1}, n_{2}, n_{3}\right)\right. \\
& \left.+\left(e_{1}^{2}+e_{2} e_{3}\right) K\left[n_{0}-1, n_{1}-1, n_{2}, n_{3}\right)\right)
\end{aligned}
$$

and its close cousins are sufficient to reduce the evaluation of monodromies of products of shifted Weierstrass functions to known integrals.

\section{The group action}

The Dynkin diagram of affine $D_{4}^{(1)}$ is depicted in figure 1 . If we delete the affine node labeled by zero, we obtain the Dynkin diagram of $D_{4}$. The outer automorphisms of $D_{4}$, i.e. triality, act by exchanging the external nodes of the $D_{4}$ Dynkin diagram. With the basis 
$\left\{\epsilon_{i}\right\}$ of root space introduced in section 2.2 , we have

$$
\begin{aligned}
\alpha_{0}^{\vee} & =\delta-\left(\epsilon_{1}+\epsilon_{2}\right), \\
\alpha_{1} & =\epsilon_{1}-\epsilon_{2}, \\
\alpha_{2} & =\epsilon_{2}-\epsilon_{3}, \\
\alpha_{3} & =\epsilon_{3}-\epsilon_{4}, \\
\alpha_{4} & =\epsilon_{3}+\epsilon_{4} .
\end{aligned}
$$

The group $\mathrm{SL}(2, \mathbb{Z})$ is the semi-direct product $\mathrm{SL}(2, \mathbb{Z})=\Gamma(2) \rtimes \mathrm{S}_{3}$. If we write elements of the set $\Gamma(2) \times S_{3}$ as pairs $(\gamma, p)$, then the group structure of $\mathrm{SL}(2, \mathbb{Z})$ is given by $\left(\gamma_{1}, p_{1}\right) \cdot\left(\gamma_{2}, p_{2}\right)=\left(\gamma_{1} a_{p_{1}}\left(\gamma_{2}\right), p_{1} p_{2}\right)$ where $a_{p_{1}}$ are the automorphisms of $\Gamma(2)$ induced by conjugation of $\Gamma(2)$ by the elements $S, S T, S T S, S T S T, S T S T S, T$ of $\mathrm{SL}(2, \mathbb{Z})$.

The full symmetry group of the theory is $\left(W\left(D_{4}\right) \times \Gamma(2)\right) \rtimes S_{3}$, where $S_{3}$ acts as outer automorphisms on $\mathrm{SO}(8)$ and by automorphisms inner to $\mathrm{SL}(2, \mathbb{Z})$ on $\Gamma(2)$. The composition in the symmetry group is $\left(g_{1}, \gamma_{1}, p_{1}\right) \cdot\left(g_{2}, \gamma_{2}, p_{2}\right)=\left(g_{1} \tau_{p_{1}}\left(g_{2}\right), \gamma_{1} a_{p_{1}}\left(\gamma_{2}\right), p_{1} p_{2}\right)$ where $\tau_{p_{1}}$ is the outer automorphism of $\mathrm{SO}(8)$ associated to the permutation element $p_{1}$ of $S_{3}$.

Specifically, the $S$ transformation permutes the vector and the spinor representation in the spectrum, while the $T$ transformation permutes the spinor and the conjugate spinor representation [6]. With the fundamental weights $\mu_{1}, \mu_{3}, \mu_{4}$, dual to the respective simple roots, corresponding to the vector, spinor, and conjugate spinor respectively, we conclude that $p_{S}$ permutes $\alpha_{1}$ and $\alpha_{3}$, while $p_{T}$ permutes $\alpha_{3}$ and $\alpha_{4}$. It can be checked that this convention is consistent with our convention for the cross ratio $x$ as a function of the modular parameter $\tau$, and the action of the modular group on the masses $M_{i}$.

Open Access. This article is distributed under the terms of the Creative Commons Attribution License which permits any use, distribution and reproduction in any medium, provided the original author(s) and source are credited.

\section{References}

[1] L.F. Alday, D. Gaiotto and Y. Tachikawa, Liouville Correlation Functions from Four-dimensional Gauge Theories, Lett. Math. Phys. 91 (2010) 167 [arXiv:0906.3219] [INSPIRE].

[2] V. Fateev and A. Litvinov, On AGT conjecture, JHEP 02 (2010) 014 [arXiv:0912.0504] [INSPIRE].

[3] A. Marshakov, A. Mironov and A. Morozov, On AGT Relations with Surface Operator Insertion and Stationary Limit of Beta-Ensembles, J. Geom. Phys. 61 (2011) 1203 [arXiv: 1011.4491] [INSPIRE].

[4] A.-K. Kashani-Poor and J. Troost, The toroidal block and the genus expansion, JHEP 03 (2013) 133 [arXiv: 1212.0722] [INSPIRE].

[5] A. Zamolodchikov, Two-dimensional conformal symmetry and critical four-spin correlation functions in the Ashkin-Teller model, Sov. Phys. JETP 63 (1986) 1061. 
[6] N. Seiberg and E. Witten, Monopoles, duality and chiral symmetry breaking in $N=2$ supersymmetric QCD, Nucl. Phys. B 431 (1994) 484 [hep-th/9408099] [INSPIRE].

[7] G. Giribet, On AGT description of $N=2$ SCFT with $N(f)=$ 4, JHEP 01 (2010) 097 [arXiv:0912.1930] [INSPIRE].

[8] A. Zamolodchikov and V. Fateev, Operator Algebra and Correlation Functions in the Two-Dimensional Wess-Zumino $\mathrm{SU}(2) \times \mathrm{SU}(2)$ Chiral Model, Sov. J. Nucl. Phys. 43 (1986) 657 [INSPIRE].

[9] V. Fateev and A. Litvinov, Multipoint correlation functions in Liouville field theory and minimal Liouville gravity, Theor. Math. Phys. 154 (2008) 454 [arXiv:0707.1664] [INSPIRE].

[10] L.F. Alday, D. Gaiotto, S. Gukov, Y. Tachikawa and H. Verlinde, Loop and surface operators in $N=2$ gauge theory and Liouville modular geometry, JHEP 01 (2010) 113 [arXiv: 0909.0945] [INSPIRE].

[11] A. Levin and Olshanetsky, Painlevé-Calogero correspondence, arXiv:alg-geom/9706012.

[12] H. Nagoya, A quantization of the sixth Painlevé equation, Adv. Stud. Pure Math. 55 (2009) 291.

[13] A. Zabrodin and A. Zotov, Quantum Painleve-Calogero Correspondence, J. Math. Phys. 53 (2012) 073507 [arXiv: 1107.5672] [INSPIRE].

[14] P. Painlevé, Sur les équations difféérentielles du second ordre et d'ordre supérieur dont l'intégrale générale est uniforme, Acta Math. 25 (1902) 185.

[15] R. Fuchs, Uber linear homogene Differentialgleichungen zweiter Ordnung mit im endlich gelegene wesentlich singularen Stellen, Math. Ann. 63 (1907) 301.

[16] B. Gambier, Sur les équations différentielles du second ordre et du premier degré dont l'intégrale générale est à points critiques fixes, CR Ac. Sci. Paris 142 (1906) 266.

[17] H. Nagoya, Realizations of affine Weyl group symmetries on the quantum Painleve equations by fractional calculus, Lett. Math. Phys. 102 (2012) 297 [InSPIRE].

[18] N.A. Nekrasov, Seiberg-Witten prepotential from instanton counting, Adv. Theor. Math. Phys. 7 (2004) 831 [hep-th/0206161] [INSPIRE].

[19] L. Schlesinger, Uber eine Klasse von Differentialsystemen beliebliger Ordnumg mit festen kritischer Punkten, J. fUr Math. 141 (1912) 96.

[20] R. Garnier, Sur des équations différentielles du troisième ordre dont l'intégrale est uniform et sur une classe d'équations nouvelles d'ordre supérieur dont l'intégrale générale a ses point critiques fixés, Ann. Sci. de l'ENS 29 (1912) 1.

[21] R. Garnier, Sur une classe de systèmes differentiels abéliens deduits de la théorie des équations linéaires, Rend. Circ. Mat. Palermo 43 (1918-19) 155.

[22] M. Jimbo and T. Miwa, Monodromy perserving deformation of linear ordinary differential equations with rational coefficients. II, Physica D 2 (1981) 407.

[23] K. Okamoto, Studies on the Painlevé equations. I. Sixth Painlevé equation, Ann. Mat. Pura Appl. (4) 146 (1987) 337.

[24] H. Nagoya and Y. Yamada, Symmetries of quantum Lax equations for the Painlevé equations, arXiv: 1206.5963 [INSPIRE]. 
[25] P. Boalch, Six results on Painlevé VI, Société Mathématique de France, Séminaires et congrès 14 (2006) 1.

[26] A. Zamolodchikov, Conformal symmetry in two-dimensional space: recursion representation of the conformal block, Theor. Math. Phys. 73 (1987) 1088.

[27] Y. Manin, Sixth Painlevé Equation, Universal Elliptic Curve, and Mirror of $\mathbb{P}^{2}, A M S$ Transl. (2) 186 (1998) 131.

[28] M. Olshanetsky and A. Perelomov, Classical integrable finite dimensional systems related to Lie algebras, Phys. Rept. 71 (1981) 313 [INSPIRE].

[29] V. Inozemtsev, Lax Representation with Spectral Parameter on a Torus for Particle Systems, Lett. Math. Phys. 17 (1989) 11.

[30] M. Gaudin, Diagonalisation d'une classe d'Hamiltoniens de spin, J. Physique 37 (1976) 1087.

[31] A. Zotov, Elliptic linear problem for Calogero-Inozemtsev model and Painleve VI equation, Lett. Math. Phys. 67 (2004) 153 [hep-th/0310260] [INSPIRE].

[32] K. Takasaki, Painleve-Calogero correspondence revisited, J. Math. Phys. 42 (2001) 1443 [math/0004118] [INSPIRE].

[33] V.A. Fateev, A. Litvinov, A. Neveu and E. Onofri, Differential equation for four-point correlation function in Liouville field theory and elliptic four-point conformal blocks, J. Phys. A 42 (2009) 304011 [arXiv:0902.1331] [INSPIRE].

[34] M. Kaneko and D. Zagier, A generalized Jacobi theta function and quasimodular forms, in The Moduli Space of Curves, Dijkgraaf, Faber, vanderGeer eds., Birkhäuser, (1995).

[35] M.-x. Huang, A.-K. Kashani-Poor and A. Klemm, The $\Omega$ deformed B-model for rigid $\mathcal{N}=2$ theories, Annales Henri Poincaré 14 (2013) 425 [arXiv:1109.5728] [INSPIRE].

[36] A.B. Zamolodchikov and A.B. Zamolodchikov, Conformal field theory and 2-D critical phenomena. 3. Conformal bootstrap and degenerate representations of conformal algebra, ITEP-90-31 (1990).

[37] A. Zamolodchikov, Conformal symmetry in two dimensions: an explicit recurrence formula for the conformal partial wave amplitude, Commun. Math. Phys. 96 (1984) 419.

[38] M. Billó, M. Frau, L. Gallot, A. Lerda and I. Pesando, Deformed $N=2$ theories, generalized recursion relations and S-duality, JHEP 04 (2013) 039 [arXiv:1302.0686] [INSPIRE].

[39] M. Billó, M. Frau, L. Gallot and A. Lerda, The exact 8d chiral ring from 4d recursion relations, JHEP 11 (2011) 077 [arXiv:1107.3691] [INSPIRE]. 